\title{
Residual function of cystic fibrosis mutants predicts response to small molecule CFTR modulators
}

\author{
Sangwoo T. Han, ${ }^{1}$ Andras Rab, ${ }^{2}$ Matthew J. Pellicore,, ${ }^{1}$ Emily F. Davis, ${ }^{1}$ Allison F. McCague, ${ }^{1}$ \\ Taylor A. Evans, ${ }^{1}$ Anya T. Joynt, ${ }^{1}$ Zhongzhou Lu, ${ }^{1}$ Zhiwei Cai, ${ }^{3}$ Karen S. Raraigh, ${ }^{1}$ Jeong S. Hong, ${ }^{2}$ \\ David N. Sheppard, ${ }^{3}$ Eric J. Sorscher, ${ }^{2}$ and Garry R. Cutting ${ }^{1}$ \\ 'McKusick-Nathans Institute of Genetic Medicine, Johns Hopkins University School of Medicine, Baltimore, Maryland, \\ USA. 'Department of Pediatrics, Emory University School of Medicine, Atlanta, Georgia, USA. ${ }^{3}$ School of Physiology, \\ Pharmacology and Neuroscience, University of Bristol, Bristol, United Kingdom.
}

Treatment of individuals with cystic fibrosis (CF) has been transformed by small molecule therapies that target select pathogenic variants in the CF transmembrane conductance regulator (CFTR). To expand treatment eligibility, we stably expressed 43 rare missense CFTR variants associated with moderate CF from a single site in the genome of human CF bronchial epithelial (CFBE410') cells. The magnitude of drug response was highly correlated with residual CFTR function for the potentiator ivacaftor, the corrector lumacaftor, and ivacaftor-lumacaftor combination therapy. Response of a second set of 16 variants expressed stably in Fischer rat thyroid (FRT) cells showed nearly identical correlations. Subsets of variants were identified that demonstrated statistically significantly higher responses to specific treatments. Furthermore, nearly all variants studied in CFBE cells (40 of 43 ) and FRT cells (13 of 16) demonstrated greater response to ivacaftor-lumacaftor combination therapy than either modulator alone. Together, these variants represent $87 \%$ of individuals in the CFTR2 database with at least 1 missense variant. Thus, our results indicate that most individuals with CF carrying missense variants are (a) likely to respond modestly to currently available modulator therapy, while a small fraction will have pronounced responses, and (b) likely to derive the greatest benefit from combination therapy.

Authorship note: STH and AR contributed equally to this work.

Conflict of interest: The authors have declared that no conflict of interest exists.

Submitted: March 22, 2018

Accepted: June 12, 2018

Published: July 25, 2018

\section{Reference information:} JCI Insight. 2018;3(14):e121159. https://doi.org/10.1172/jci. insight.121159.

\section{Introduction}

Targeting specific mutant forms of defective cystic fibrosis transmembrane conductance regulator (CFTR) with orally bioavailable modulators has revolutionized treatment of CF (1-4). Ivacaftor, the first drug to achieve US Food and Drug Administration (FDA) approval, potentiates function by altering chloride channel gating and increasing the open probability $\left(\mathrm{P}_{\mathrm{o}}\right)$ of CFTR (5-7). Ivacaftor was originally approved for individuals with CF who carried at least 1 copy of the p.Gly551Asp (G551D) variant. Subsequent clinical trials demonstrated benefit for individuals with CF who carried at least 1 copy of any 1 of 8 variants that affected the gating of CFTR $(8,9)$. The second drug, lumacaftor, was developed to correct protein folding defects caused by the most common CF-causing variant, p.Phe508del (F508del) (10, 11). While lumacaftor alone was not clinically effective for individuals homozygous for F508del (12), coadministration of lumacaftor and ivacaftor improved clinical outcome measures for individuals homozygous for F508del (13). Recently, tezacaftor, a second corrector compound, demonstrated clinical efficacy in F508del homozygotes when combined with ivacaftor (14). The same combination of CFTR-targeted drugs also proved efficacious in a clinical trial of individuals with CF who carried F508del in trans with a select set of residual function variants (15).

Ivacaftor alone or in combination with either lumacaftor or tezacaftor has demonstrated efficacy for 39 of the approximately 1,640 CFTR variants associated with CF (http://www.CFTR2.org). This leaves thousands of individuals with $C F$ who carry variants that have not been approved or in many cases even experimentally tested for response to these 3 drugs. Review of the untested variants indicates that approximately $50 \%$ are predicted to generate CFTR protein and, therefore, could potentially be targeted with the currently available drugs (3). Unfortunately, clinical trials of uncommon variants are difficult to conduct due to the wide geographic dispersion of the small number of individuals carrying these variants. Moreover, the high 
cost of CFTR modulators has made off-label prescription problematic. Even if an individual with a rare variant responds well in the clinic, insurers may not support the cost of treatment unless the modulator is FDA approved for that particular genotype. Thus, alternative approaches are needed to assess the response to CFTR modulators for rare variants. Cell-based functional assays represent an avenue for evaluating rare variants in cases where clinical studies or assessment of primary tissues are impractical, provided these systems are well vetted and generate reproducible results. Fischer rat thyroid (FRT) cells have been extensively used as a model cell line for studying the role of CFTR in epithelial ion transport $(16,17)$, and FRT cell lines expressing CFTR cDNA have been used in a number of studies to generate response data that have provided preliminary evidence to proceed to clinical trials $(5,10,18,19)$ and, more recently, to facilitate drug label expansion (20). CF bronchial epithelial cell line CFBE41o- (herein referred to as CFBE) cells provide an opportunity to test the effects of CFTR variants in a human cell line from a relevant tissue type with a transcriptome that is very similar to that of primary airway epithelial cells (21). These 2 cell lines offer complementary platforms to evaluate the functional consequences and responses to modulators of CFTR missense variants in a standardized and reproducible manner.

In this study, we utilized CFBE cells stably expressing CFTR missense variants to extend our understanding of drug responses to $C F T R$ bearing rare (minor allele frequency $[\mathrm{MAF}]<1 \%$ in the CF population) missense variants. Our initial goal was to identify variants with either positive or less favorable responses to ivacaftor, lumacaftor, or ivacaftor-lumacaftor combination treatment to inform clinical applications. However, we discovered that response to the modulators was closely correlated with residual function of the mutant forms of CFTR for most variants expressed in CFBE cells. This observation was replicated with a different set of missense variants expressed stably in FRT cells and was also apparent upon retrospective analysis of previously published ivacaftor studies using another independent set of FRT cells (18). Using these results, we devised a statistically valid approach to identify robust responders to ivacaftor and lumacaftor based on the fold change in CFTR function. Furthermore, we showed that the combination of the 2 modulators produces a greater response for most missense variants, including high-response variants, than either drug alone. These observations, in concert with the recent demonstration that combinatorial treatment was efficacious for residual function variants (15), suggest that CFTR-targeted treatment may be appropriate for most individuals with CF carrying residual function missense variants.

\section{Results}

To assess the response of CFTR variants associated with a wide range of CF phenotypes to FDA-approved CFTR modulators, we studied 57 missense variants reported in individuals with CF using 2 cellular expression systems. Forty-three rare missense variants that were associated with a range of phenotypes measured by modest increases in sweat chloride concentration (40-90 mM) and/or pancreatic exocrine sufficiency (PS) prevalence greater than 50\% were selected from the CFTR2 database (Table 1) and reported previously (22). Due to the moderate pancreatic disease, each variant was expected to allow residual CFTR function $(23,24)$ and was associated with less severe disease. To approximate the native environment of CFTR in the lung, the rare variants were studied in CBFE cells that are devoid of endogenous CFTR expression $(21,25)$. These CFBE cells have been used to generate cell lines expressing CFTR missense variants at a level comparable with primary human tissues in order to measure their effect on CFTR function and aid in interpretation of disease liability (22). A second set of 16 variants were expressed in FRT cells (Table 2), a cell type that has been employed to evaluate the response of CFTR to FDA-approved drugs (20), although the studies presented here were performed on cell lines selected within a more narrow range of mRNA expression. These variants are generally more frequent in individuals with CF and are associated with more severe disease $(60-106 \mathrm{mM}$ and $2 \%-80 \%$ PS). Two variants, p.Arg334Trp (R334W) and p.Thr338Ile (T338I), were common to both groups, yielding a total study set of 57 unique variants. Variants F508del and G551D, which have been extensively studied previously, were also analyzed in both cell lines (3), bringing the total number of cell lines studied here to 63.

In each cell system, CFTR bearing each of the missense variants was stably expressed from a single site of integration, as previously described $(18,21,22,26)$. Residual CFTR function of each missense variant in CFBE cells was measured as the magnitude of forskolin-mediated $(10 \mu \mathrm{M}) \mathrm{cAMP}$-stimulated current inhibited by CFTR $_{\text {inh }}-172$ (inh-172; $10 \mu \mathrm{M}$ ), a widely used potent inhibitor of CFTR (27), normalized to WT function based on mRNA expression level, as previously described (22), to calculate a precise percent-WT function. CFTR function in FRT cells was measured as the magnitude of forskolin-mediated $(5 \mu \mathrm{M})$ cAMP-stimulated current (with specificity confirmed by inh-172 [10 $\mu \mathrm{M}]$ ) 
Table 1. Measurements of CFTR function and response to modulators in CFBE cells

\begin{tabular}{|c|c|c|c|c|c|c|}
\hline Variant & HGVS & cDNA & Residual function ( $n$ ) & $\begin{array}{c}10 \mu \mathrm{M} \\
\text { Ivacaftor (n) }\end{array}$ & $\begin{array}{c}6 \mu \mathrm{M} \\
\text { Lumacaftor (n) }\end{array}$ & $\begin{array}{l}10 \mu \mathrm{M} \text { ivacaftor } 6 \\
\mu \mathrm{M} \text { lumacaftor }(n)\end{array}$ \\
\hline P5L & p.Pro5Leu & c. $14 C>T$ & $21.9 \pm \pm 2(3)$ & $25.9 \pm 0.6(3)$ & $57.72 \pm 0.6(3)$ & $62.2 \pm 1.4(3)$ \\
\hline G27R & p.Gly27Arg & c.79G $>A$ & $0.9 \pm 0.1(3)$ & $0.8 \pm 0(3)$ & $5.14 \pm 0.1(3)$ & $5.2 \pm 0.3(3)$ \\
\hline W57C & p.Trp57Cly & c. $169 \mathrm{~T}>\mathrm{G}$ & $1.1 \pm 0.1(3)$ & $1.3 \pm 0.2(3)$ & $1.89 \pm 0.1(3)$ & $2.5 \pm 0.1(3)$ \\
\hline G91R & p.Gly91Arg & c. $271 G>A$ & $1.6 \pm 0.2(3)$ & $1.7 \pm 0.1(3)$ & $14.29 \pm 0.3(3)$ & $21.1 \pm 0.2(3)$ \\
\hline Q98R & p.Gln98Arg & c. $293 A>C$ & $4.5 \pm 0.1(3)$ & $5.5 \pm 0.2(3)$ & $14.32 \pm 0.5(3)$ & $18.8 \pm 0.9$ (3) \\
\hline $\mathrm{R} 117 \mathrm{~L}$ & p.Arg117Leu & c.350G $>\mathrm{T}$ & $10.2 \pm 2.5$ (3) & $17.8 \pm 1.3(3)$ & $13.2 \pm 1.5(3)$ & $25.4 \pm 1.3(3)$ \\
\hline L138ins & p.Leu138insLeu & c.413_415dupTAC & $1.6 \pm 0.2(3)$ & $2.2 \pm 0.2(3)$ & $16.1 \pm 0.9(3)$ & $29.8 \pm 1.1(3)$ \\
\hline H139R & p.His139Arg & c. $416 A>C$ & $1.5 \pm 0.5(3)$ & $2.3 \pm 0.4(3)$ & $3.0 \pm 0.3(3)$ & $3.3 \pm 0.9(3)$ \\
\hline $\mathrm{L} 145 \mathrm{H}$ & p.Leu145His & c. $434 \mathrm{~T}>\mathrm{A}$ & $5.6 \pm 0.4(3)$ & $7.5 \pm 0.4(3)$ & $51.5 \pm 5.4(3)$ & $72.8 \pm 3.6(3)$ \\
\hline L165S & p.Leu165Ser & c.494T>C & $1.9 \pm 0.1(3)$ & $1.7 \pm 0.1(3)$ & $3.1 \pm 0.2(3)$ & $4.8 \pm 0.1(3)$ \\
\hline R334Q & p.Arg334GIn & c.1001G>A & $25.5 \pm 1.4(29)$ & $34.2 \pm 2.3(30)$ & $30.2 \pm 2.1(15)$ & $40.9 \pm 2.3(15)$ \\
\hline I336K & p.lle336Lys & c.1007T>A & $2.3 \pm 0.3(20)$ & $3.2 \pm 0.4(24)$ & $9.2 \pm 0.9(13)$ & $20.1 \pm 1.5(26)$ \\
\hline T338I & p.Thr338lle & c. $1013 C>T$ & $6.4 \pm 0.3(18)$ & $14.2 \pm 0.8(18)$ & $8.9 \pm 0.8$ (9) & $20.3 \pm 1.8(9)$ \\
\hline I340N & p.lle340Asn & c.1019T>A & $12.5 \pm 0.9(33)$ & $16.5 \pm 1.6(21)$ & $70 \pm 10.4(20)$ & $79.8 \pm 17.7$ (14) \\
\hline S341P & p.Ser341Pro & c. $1021 T>C$ & $0.8 \pm 0.1(11)$ & $1.5 \pm 0.1(13)$ & $1.2 \pm 0.1(11)$ & $2.9 \pm 0.2(13)$ \\
\hline M348V & p.Met348Val & c. $1042 A>C$ & $77.8 \pm 7.4$ (6) & $138.8 \pm 7.3(6)$ & $103.0 \pm 17.3$ (3) & $124.2 \pm 56.3(6)$ \\
\hline A349V & p.Ala349Val & c. $1046 C>T$ & $45.5 \pm 4.7(13)$ & $110.7 \pm 11.2(14)$ & $94.9 \pm 16.3(7)$ & $139.5 \pm 32.3(11)$ \\
\hline L453S & p.Leu453Ser & c. $1358 \mathrm{~T}>C$ & $3.4 \pm 0.2(3)$ & $3.6 \pm 0.3(3)$ & $6.7 \pm 0.3(3)$ & $7.6 \pm 0.1(3)$ \\
\hline V456A & p.Val456Ala & c. $1367 T>C$ & $4.4 \pm 0.4(3)$ & $6.9 \pm 0(3)$ & $8.2 \pm 0.7$ (3) & $11.5 \pm 0.4(3)$ \\
\hline E474K & p.Glu474Lys & c. $1420 G>A$ & $1.2 \pm 0.1(3)$ & $1 \pm 0.1(3)$ & $3.9 \pm 0.5(3)$ & $5.9 \pm 0.2(3)$ \\
\hline F508del & p.Phe508del & c.1521_1523del & $0.5 \pm 0.1(15)$ & $0.9 \pm 0.2(11)$ & $1.8 \pm 0.5(10)$ & $3.2 \pm 0.7(10)$ \\
\hline D979V & p.Asp979Val & c. $2936 \mathrm{~A}>\mathrm{T}$ & $8.6 \pm 1.8$ (3) & $19 \pm 0.9(3)$ & $24.1 \pm 5.9(3)$ & $72.4 \pm 6.4(3)$ \\
\hline A1006E & p.Ala1006Glu & c. $3017 C>A$ & $3.9 \pm 0.4$ (3) & $4.8 \pm 0.9(3)$ & $6.4 \pm 1.4(3)$ & $10.2 \pm 0.5(3)$ \\
\hline F1016S & p.Phe1016Ser & c. $3047 \mathrm{~T}>C$ & $15.8 \pm 1.5(3)$ & $24.7 \pm 0.7(3)$ & $38.2 \pm 4.7(3)$ & $55.4 \pm 1.3(3)$ \\
\hline Y1032C & p.Tyr1032Cys & c. $3095 \mathrm{~A}>\mathrm{G}$ & $13 \pm 1.6(3)$ & $14.2 \pm 0.4(3)$ & $78.5 \pm 8.3(3)$ & $76.4 \pm 5.5(3)$ \\
\hline W1098C & p.Trp1098Cys & c.3294C>C & $3.4 \pm 0.3(6)$ & $4.1 \pm 0.3(6)$ & $12.1 \pm 0.7(6)$ & $19.1 \pm 0.9(6)$ \\
\hline $\mathrm{S} 1118 \mathrm{~F}$ & p.Ser1118Phe & c. $3353 C>T$ & $4.8 \pm 0.5(3)$ & $8.6 \pm 0.3(3)$ & $8.9 \pm 0.2(3)$ & $18.3 \pm 0.4(3)$ \\
\hline S1159F & p.Ser1159Phe & c. $3476 C>T$ & $7.4 \pm 1.3(3)$ & $43.8 \pm 2.4(3)$ & $18.4 \pm 3.8(3)$ & $59.5 \pm 2.0$ (3) \\
\hline S1159P & p.Ser1159Pro & c. $3475 \mathrm{~T}>\mathrm{C}$ & $6.4 \pm 0.5(4)$ & $29.3 \pm 1.3(4)$ & $10.9 \pm 1.6(4)$ & $42.2 \pm 1.4(4)$ \\
\hline T1246I & p.Thr1246Ile & c. $3737 C>T$ & $20 \pm 2(3)$ & $52.9 \pm 1.8$ (3) & $35.2 \pm 1.8$ (3) & $74.7 \pm 2.7$ (3) \\
\hline $\mathrm{R} 1283 \mathrm{M}$ & p.Arg1283Met & c.3848G >T & $10.3 \pm 0.9(3)$ & $12.5 \pm 0.7(3)$ & $12.1 \pm 1$ (3) & $19.1 \pm 0.7(3)$ \\
\hline L1335P & p.Leu1335Pro & c. $4004 \mathrm{~T}>\mathrm{C}$ & $2.6 \pm 0.1(3)$ & $3.5 \pm 0.1$ (3) & $3.2 \pm 0.1(3)$ & $4.4 \pm 0.1(3)$ \\
\hline
\end{tabular}

HGVS, Human Genome Vairation Socienty nomenclature.

compared with WT. All FRT cell lines selected for study expressed CFTR mRNA within a narrow range (0.5- to 1.5-fold) of CFTR mRNA compared with a WT-expressing line generated by the same method (26). The 4 variants studied in both CFBE and FRT cells (R334W, T338I, F508del, and G551D) produced comparable levels of CFTR function: F508del (CFBE, 0.5\% WT; FRT, 1.6\% WT); G551D (CFBE, 3.2\% WT; FRT, 4.0\% WT); R334W (CFBE, 2.0\% WT; FRT, 3.9\% WT); T338I (CFBE, 6.4\% WT; FRT, 7.6\% WT) (Table 1 and Table 2). 
Table 2. Measurements of CFTR function and response to modulators in FRT cells

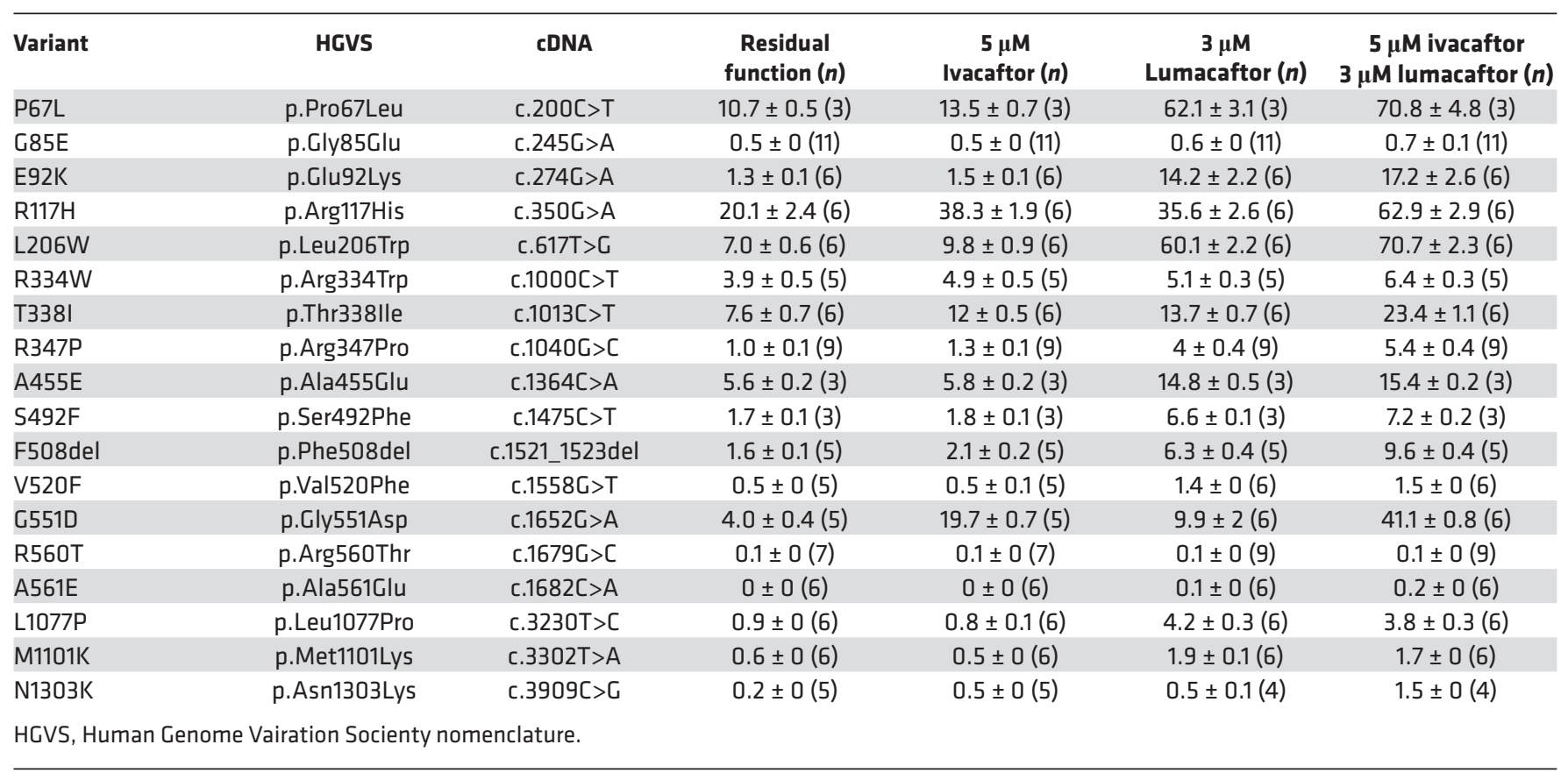

CFTR missense variants expressed in CFBE cells were first tested for their response to $10 \mu \mathrm{M}$ ivacaftor, a compound that is efficacious for variants that affect channel gating (5). G551D-CFTR in CFBE cells demonstrated a dose-dependent response to ivacaftor from $0.1-100 \mu \mathrm{M}$, similar to previously reported findings in FRT cells (5) (data not shown). Ivacaftor enhanced the forskolin-stimulated (10 $\mu \mathrm{M})$ function of many of the variants tested in CFBE cells, regardless of whether or not they were known to impair CFTR channel gating. Furthermore, the magnitude of ivacaftor-enhanced CFTR activity correlated with the magnitude of forskolin-activated CFTR activity $(r=0.96)$ (Figure 1A, Table 1, Supplemental Table 1; supplemental material available online with this article; https://doi.org/10.1172/jci.insight.121159DS1). Data were plotted on a logarithmic scale to visualize the entire functional range of variants studied. To determine whether the observed correlation between ivacaftor response and residual function was cell-line independent, we analyzed the ivacaftor response of 16 CFTR variants and the G551D and F508del variants stably expressed in FRT cells. The response to ivacaftor also correlated with forskolin-activated CFTR function for all 18 variants expressed in FRT cells $(r=0.93)$ (Figure 1B, Table 2).

When data from CFBE and FRT studies were combined and evaluated as fold change over baseline function, 4 cell lines (G551D expressed in both CFBE and FRT cells, p.Ser1159Phe [S1159F], and p.Ser1159Pro [S1159P] in CFBE cells) were found to be outliers (defined here as greater than 2 SD above the mean of all variants tested) and labeled as high response. G551D is the prototype for highly responsive gating variants $(5,28)$, while $\mathrm{S} 1159 \mathrm{~F}$ and $\mathrm{S} 1159 \mathrm{P}$ are 2 previously uncharacterized variants. When these outliers were removed, mean and SD were recalculated for all remaining data, and 4 outliers from this analysis (p.Phe311Leu [F311L], p.Met348Val [M348V], p.Thr1246Ile [T1246I], and p.Asn1303Lys [N1303K]) were separated and labeled as intermediate response; all other variants were labeled as modest-response variants, which accounted for the vast majority of variants (Figure 1C). F311L, M348V, and T1246I are 3 previously untested variants, while N1303K is a variant of particular interest. Single-channel studies revealed that, once delivered to the plasma membrane, N1303K-CFTR exhibited a severe gating defect characterized by infrequent brief channel openings reminiscent of the G551D variant (Supplemental Figure 1A) $(29,30)$. Ivacaftor $(1 \mu \mathrm{M})$ modestly enhanced $\mathrm{P}_{\mathrm{o}}$ of the N1303K variant by increasing the frequency and duration of channel openings (Supplemental Figure 1, B-E). Taken together, the short-circuit current $\left(\mathrm{I}_{\mathrm{sc}}\right)$ and single-channel studies indicate that ivacaftor can elicit an increase in N1303K function, although the extremely low residual function prevents a large absolute change in CFTR function after ivacaftor treatment. 

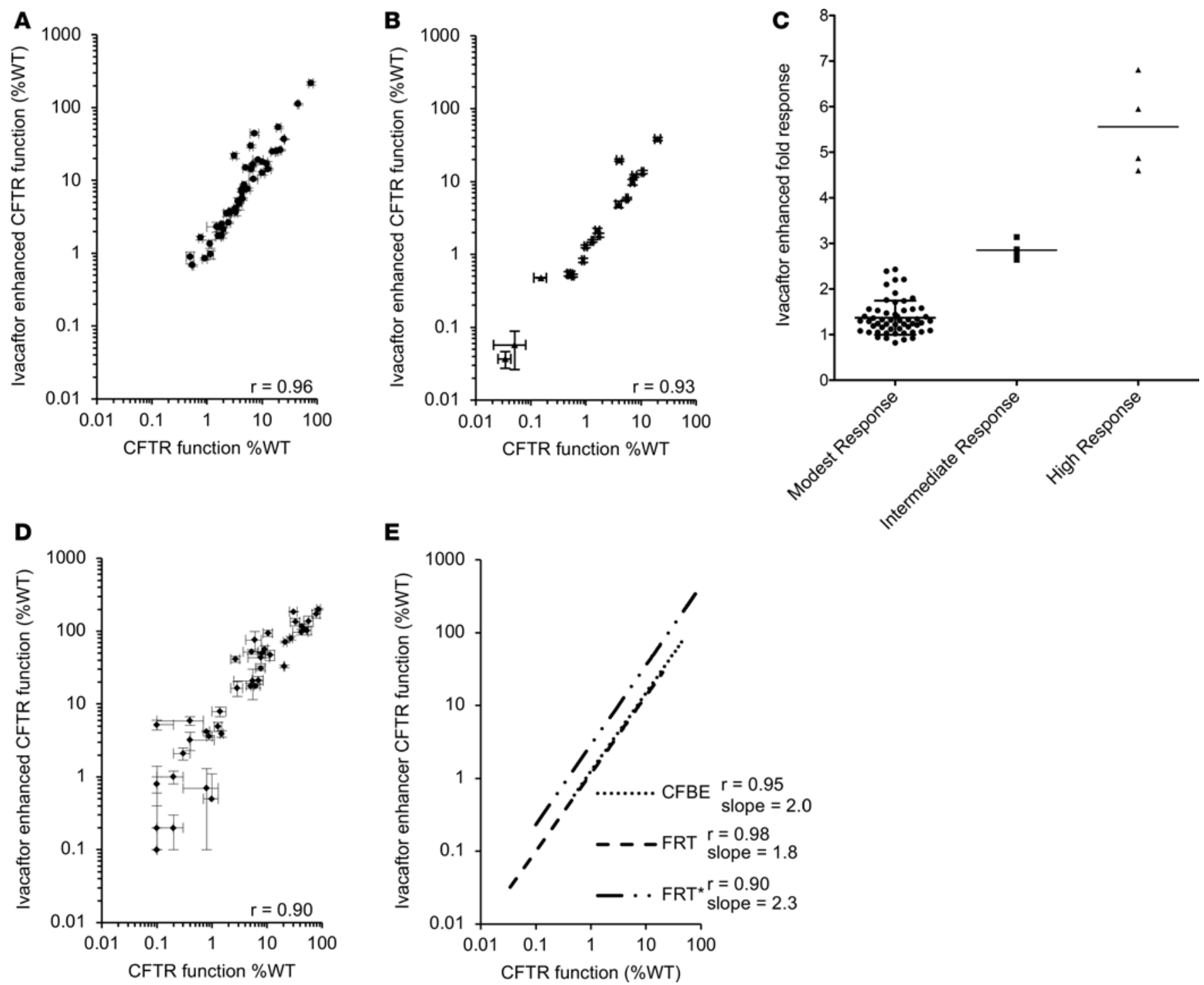

Figure 1. Ivacaftor response correlates with residual function. (A) Ivacaftor $(10 \mu \mathrm{M})$ enhanced CFTR function compared with residual forskolin-stimulated (10 $\mu M$ ) CFTR function for 45 variants expressed in CF bronchial epithelial (CFBE) cells. Each variant measured $n \geq 3$ and was plotted as mean \pm SEM on both axes. (B) Ivacaftor $(5 \mu \mathrm{M})$ enhanced CFTR function compared with residual forskolin-stimulated (5 $\mu \mathrm{M})$ CFTR function for 18 variants expressed in Fisher rat thyroid (FRT) cells. Each variant measured $n \geq 3$ and was plotted as mean \pm SEM on both axes. (C) Separation of variants based on their fold response to ivacaftor. Response of cell lines expressing G551D (CFBE), G551D (FRT), S1159F, and S1159P were designated as outliers by demonstrating fold response greater than 2 $\mathrm{SD}$ beyond the mean fold response of all variants studied in CFBE and FRT cells and labeled as high-response variants. Intermediate-response variants were those that remained outliers when high-response variants were removed from the comparison. All remaining variants were classified as modest response. Lines through data points represent the mean value $\pm 1 \mathrm{SD}$ for modest-response variants and mean of intermediate- and high-response variants. (D) Previously published data collected from FRT ${ }^{*}$ cells $(18,19)$ of nongating variants plotted as in $\mathbf{A}$ and $\mathbf{B}$. (E) Comparison of trend lines of modest-response variants identified in CFBE and FRT studies compared with nongating variants identified in FRT* ${ }^{*}$ cells. Correlation ( $r$ ) values calculated using Pearson linear correlation.

We next evaluated previously published ivacaftor response data generated by 54 CFTR variants stably expressed in a separate FRT cell line established by Vertex Pharmaceuticals (noted here as FRT $^{*}$ ) that overlapped with the 16 variants expressed in FRT cells in this study (18). Experimental conditions for measuring CFTR function were comparable with those used for the CFBE (21) and FRT (18) stable cell lines studied here. Correlation was evident for the variants studied in the FRT* cell lines (Figure 1D). Although drug doses differed between data sets, the CFBE and FRT data from this study were normalized to cells expressing WT CFTR treated with the same drug doses, allowing for comparison across cell types. When high- and intermediate-response variants were removed from analysis, the regression functions for all 3 independent studies had very similar slopes, intercepts, and high correlation (Figure 1E). These results indicate that ivacaftor increases CFTR function for most 
variants, regardless of the specific molecular defect, as the variants studied confer a range of defects in CFTR function.

Overall, the variants shared between studies yielded the same quality of ivacaftor response across systems (i.e., high-response variants in one system were high-response variants in other systems). The fold increase in current following ivacaftor treatment was similar in both cell lines for F508del (1.76-fold in CFBE and 1.31-fold in FRT), G551D (6.81 -old in CFBE and 6.02-fold in FRT), R334W (1.03-fold in CFBE and 1.24-fold in FRT), and T338I (2.21-fold in CFBE and 1.58-fold in FRT).

Identification of variants that allow CFTR to fold to a mature form but cause it to be poorly responsive to ivacaftor could be informative for future drug design and optimization. To screen for potential poor responders, we plotted the published folding and chloride channel function effects of 54 CFTR variants $(18,19)$ (Figure 2A). We noted that a group of variants, p.Ile336Lys (I336K), T338I, p.Ser341Pro (S341P), R334W, and p.Arg347Pro (R347P), located in transmembrane segment 6 (TM6) in membrane-spanning domain 1 (MSD1) of CFTR permitted partial folding but severely disrupted channel function. TM6 is notable for its role in ion conductance (31), as it is predicted to line the channel pore by homology models and cryoelectron microscopy structures (32-37), measurements of conductance properties (38-40), and evaluation of solvent accessibility (41-44). Based on these data, we hypothesized that variants that alter residues lining the channel pore might be resistant to CFTR potentiators, such as ivacaftor $(18,19)$. The 9 naturally occurring TM6 variants generated detectable mature (band C) CFTR, which suggested that each variant may allow at least partial CFTR maturation and residual CFTR function (Figure 2B; see complete unedited blots in the supplemental material). Furthermore, the ivacaftor response of TM6 variants increased as residual function increased from $<1 \% \mathrm{WT}$ to $78 \% \mathrm{WT}$, indicating that the 2 measurements are correlated (Figure 2C). The responses of the TM6 variants to ivacaftor were modest and not different from the total set of modest-response variants (Figure 2D). Furthermore, 2 TM6 variants, p.Arg334Gln (R334Q) and T338I, responded very similarly to a separate collection of 10 CFTR potentiator compounds obtained from CF Foundation Therapeutics (CFFT) CFTR Chemical Compound Program (Supplemental Figure 2). Although TM6 variants had minimal residual CFTR function despite some of them having efficient protein folding, a characteristic shared with gating variants, they are distinct in that they generated a modest response to potentiators, which was correlated with residual function similar to nongating missense variants.

Our next step was to test the response of the missense variants to the CFTR corrector lumacaftor. Lumacaftor improves the biogenesis of CFTR protein and has been primarily targeted to the common misfolding variant F508del (10). Importantly, lumacaftor has been reported to increase the function of WT CFTR and a few missense variants $(45,46)$, as well as protein folding of several other variants $(47,48)$. Accordingly, we tested the effect of lumacaftor on the variants studied here. As with ivacaftor, the magnitude of forskolin-stimulated CFTR function following incubation with $6 \mu \mathrm{M}$ lumacaftor correlated with the forskolin-stimulated function of each variant when incubated with an equal volume of DMSO for the 45 variants studied in CFBE cells (Figure 3A, Table 1). Correlation between residual function and lumacaftor response was also observed in 18 variants expressed in FRT cell lines (Figure 3B, Table 2). Like the ivacaftor response, data from CFBE and FRT cells were combined and evaluated as fold response. Outliers for the lumacaftor response were determined by measuring 2 SD above the mean; p.Gly91Arg (G91R), p.Glu92Lys (E92K), p.Leu138insLeu (L138ins), p.Leu145His (L145H), and p.Leu206Trp (L206W) were designated as high-response variants, while p.Gly27Arg (G27R), p.Pro67Leu (P67L), p.Ile340Asn (I340N), and p.Tyr1032Cys (Y1032C) were designated as intermediate-response variants (Figure 3C). When highand intermediate-response variants were removed from the analysis, the regression functions for CFBE and FRT modest-response variants demonstrated robust correlation (CFBE, $r=0.95$; FRT, $r=0.98$ ) with similar slopes (Figure 3D). These results indicate that lumacaftor increases CFTR function for most variants, regardless of the specific molecular defect, as the 59 variants studied confer a range of folding defects in CFTR from mild to severe.

Lumacaftor has been shown to be effective at improving the protein processing of variants located in MSD1 (47) and the intracellular loops (ICLs) (48). We were intrigued that 2 variants (I336K and I340N) located in TM6 within MSD1 demonstrated elevated responses to lumacaftor, which prompted further studies to verify and explain the responses of these 2 TM6 variants. Lumacaftor increased the total amount of mature CFTR protein expressed in stable CFBE cell lines for all variants (Figure 4A; see complete unedited blots in the supplemental material), even though many of them are naturally fully folded and processed (note the absence of immature band $\mathrm{B}$ protein for most variants). We confirmed that this increase in protein 
A

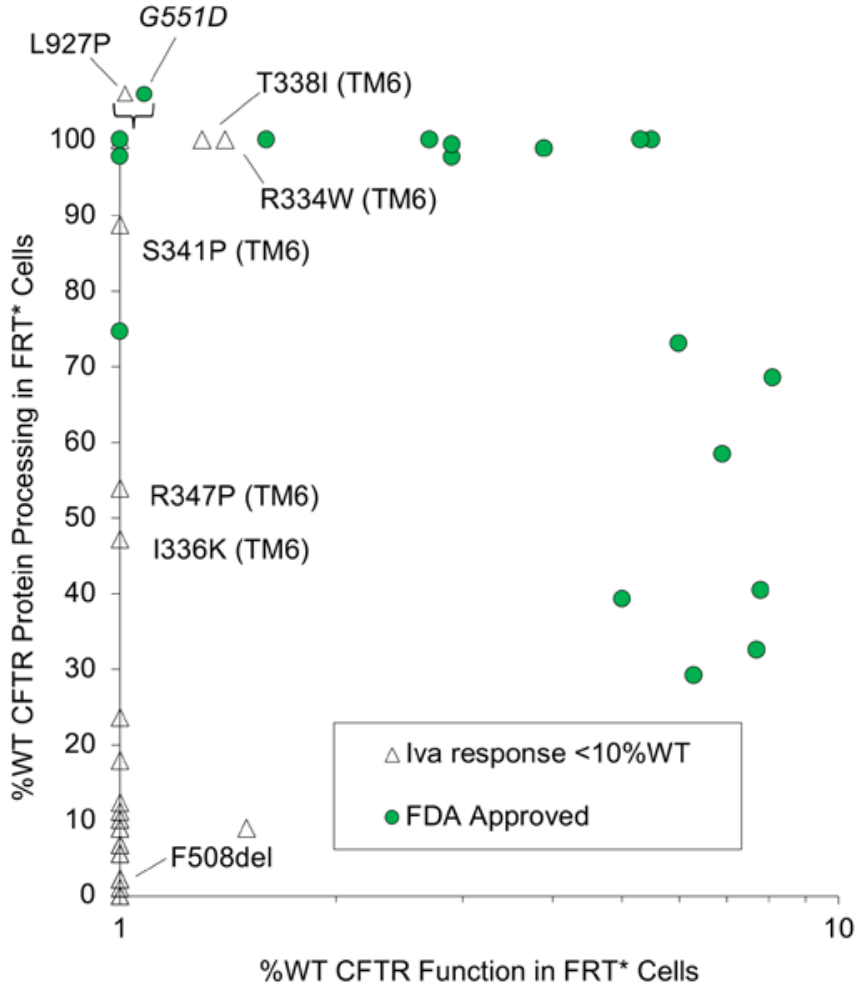

B
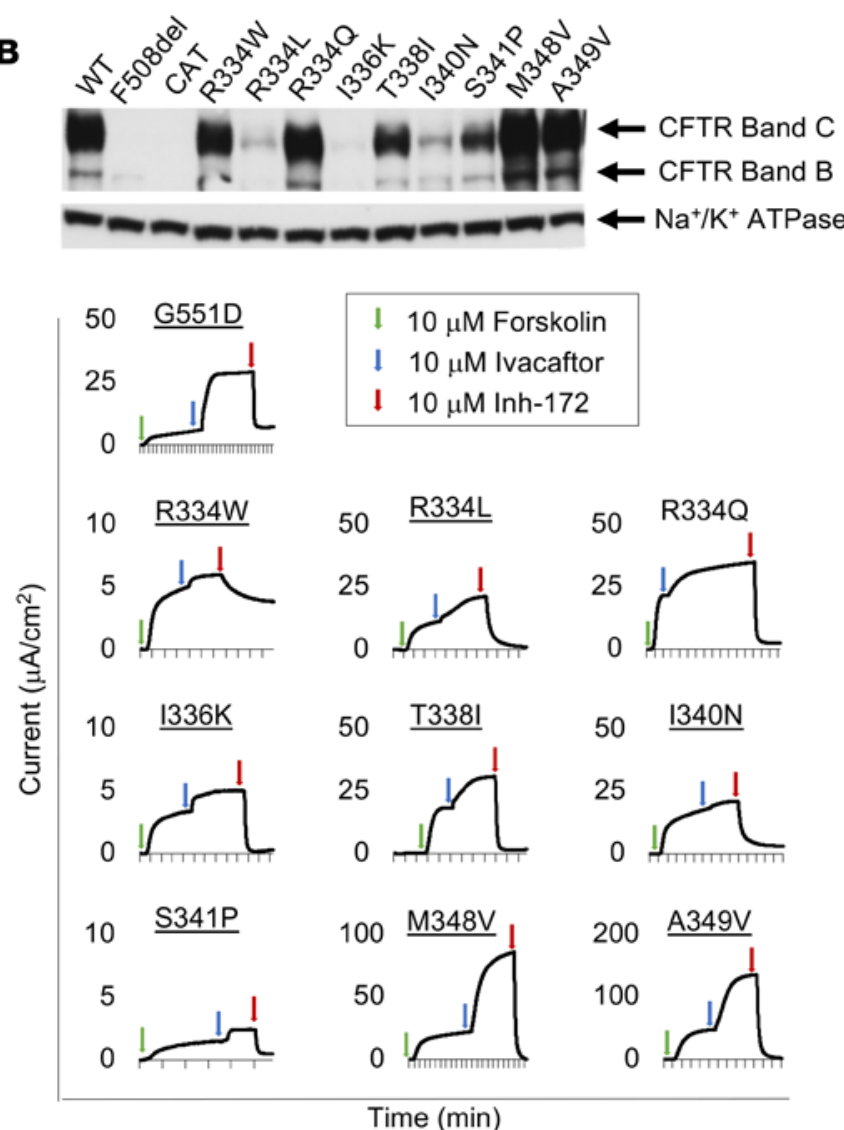
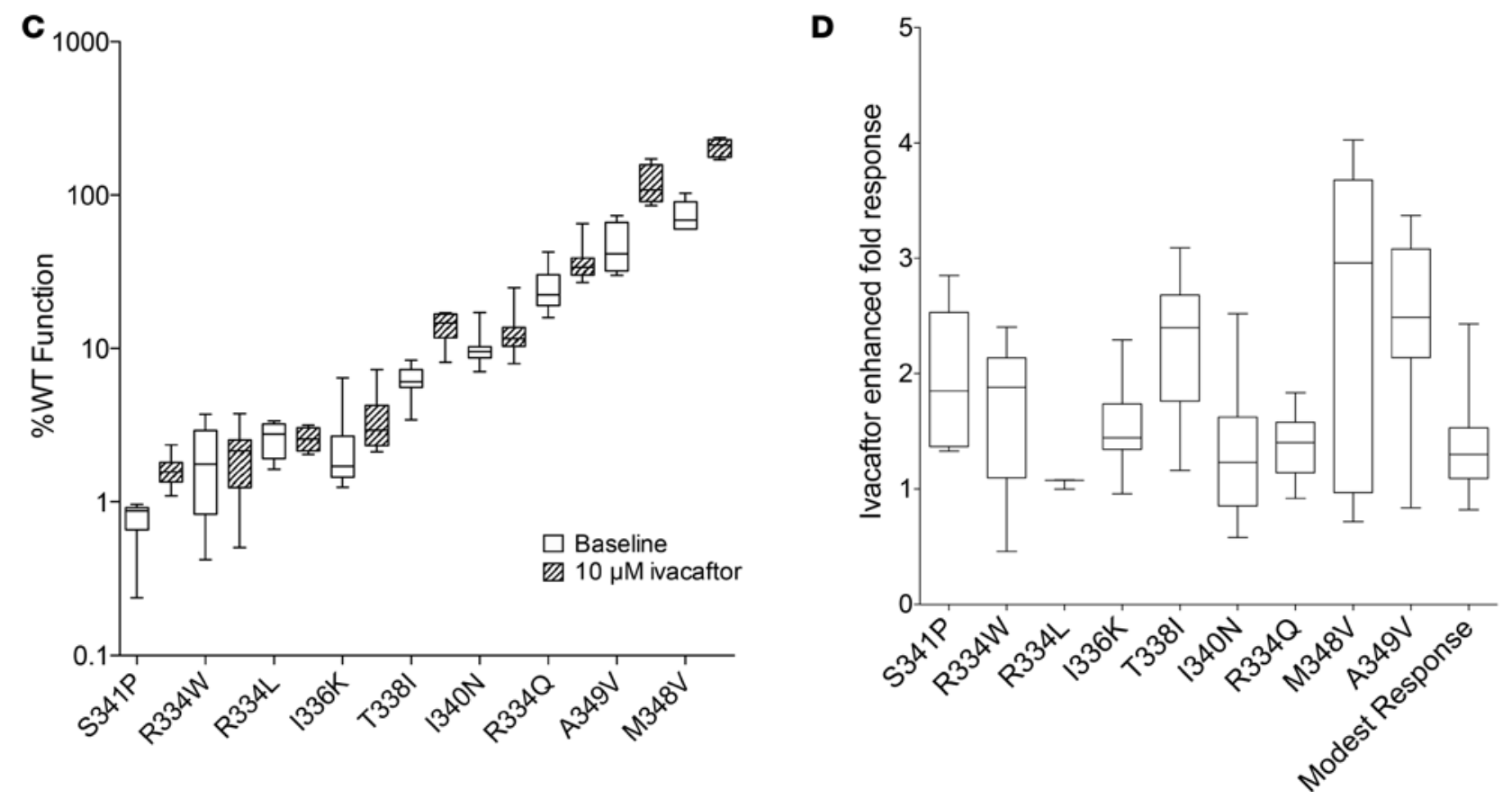

Figure 2. Variants located in the sixth transmembrane domain (TM6) show modest response to ivacaftor. (A) Plot of CFTR processing versus residual function for 54 variants previously expressed in FRT* cells (18) reveals heterogeneous response to $10 \mu \mathrm{M}$ ivacaftor of partially or well-processed low-residual function missense variants (labeled). Filled green circles represent variants approved by FDA for ivacaftor treatment; G551D labeled in italics. (B) Western blot demonstrating that all TM6 variants produce mature C band CFTR protein when transiently expressed in HEK293 cells and representative $I_{\mathrm{sc}}$ tracings of all TM6 variants stably expressed in CF bronchial epithelial (CFBE) cells demonstrating response to acute treatment with $10 \mu \mathrm{M}$ ivacaftor, recorded as area-corrected current $\left(\mu \mathrm{A} / \mathrm{cm}^{2}\right)$, over time, measured in minutes represented by tick marks in 1-minute intervals. Data are representative of $n \geq 3$ for each variant. (C) Summary data for response of TM6 variants to acute treatment with $10 \mu \mathrm{M}$ ivacaftor expressed as \%WT function. Box plots divide the data by quartile, with the median value indicated by a horizontal line within the box and whiskers extended to minimum 
and maximum values. (D) Fold response for acute treatment with $10 \mu \mathrm{M}$ ivacaftor calculated over residual function (10 $\mu \mathrm{M}$ forskolin) of TM6 variants compared with modest-responsive variants identified in this study. Box plots divide the data by quartile, with the median value indicated by a horizontal line within the box and whiskers extended to minimum and maximum values.

quantity was not due to an increase in CFTR mRNA (Supplemental Figure 3). Given the variability in the fold response to lumacaftor (Figure 3, A and B), we tested up to 4 independently derived CFBE cell lines stably expressing each of the 9 TM6 variants (Figure 4B). Multiple measurements of the different clones of TM6 variants demonstrated that the response to lumacaftor becomes more pronounced at higher levels of residual function (Figure 4C). Notably, 2 TM6 variants (I336K and I340N) had significantly higher fold responses than the remaining 7 TM6 variants (Figure 4D). To explore whether the less responsive TM6 variants respond to other CFTR correctors, we evaluated R334Q and T338I alongside the highly responsive variant I340N using a series of 18 compounds obtained from the CFFT CFTR Chemical Compound Program. Only 2 compounds generated a greater response compared with the vehicle control (DMSO) for all 4 variants tested (C17 and C18), but neither approached the efficacy of lumacaftor (Supplemental Figure 4). Since the TM6 variants are located on an $\alpha$ helix that spans the cell membrane, we speculated that the robust corrector activity might be related to the orientation of the side chains of the mutated residues relative to the proposed ion pore. Using the predicted $\alpha$ helical structure of TM6 when CFTR is in its open conformation, the side chains of residues R334, T338, S341, and M348 are predicted to reside in the channel pore, while I336, I340, and A349 are oriented such that they are embedded in either the protein or the lipid bilayer (Figure 4E). The results of arginine scanning mutagenesis are consistent with this orientation for these residues (49), as are the cryo-EM structures of CFTR in the ATP-bound state (37). Grouping of the responses of TM6 variants by predicted orientation revealed a significant correlation with lumacaftor response (Figure 4F). These results suggest that amino acid substitutions at embedded residues in TM6 are particularly responsive to lumacaftor.

The combination therapy of ivacaftor and lumacaftor (iva/lum) and ivacaftor paired with a different corrector, tezacaftor, has demonstrated clinical benefit in individuals homozygous for F508del (13, 14), and the latter combination is effective for individuals carrying 1 copy of F508del and a variant that allows partial CFTR function (15). Based on these observations and the responses we observed with potentiator and corrector alone, we sought to determine the effects of acute administration of ivacaftor after incubation (24 hours in CFBE cells or 48 hours in FRT cells) with lumacaftor (iva/ lum) on the 59 variants tested here. The response of 45 variants stably expressed in CFBE cells to iva/ lum correlated with CFTR function without modulators (Figure 5A). Results obtained from 18 variants expressed in FRT cells demonstrated a similar relationship (Figure 5B). High-response and intermediate-response variants were separated from modest-response variants (Figure 5C); G91R, E92K, L138ins, L145H, and G551D (CFBE) were designated high-response variants, while L206W, I336K, G551D, and N1303K were designated as intermediate-response variants (note that absolute magnitude of N1303K activity is quite low, although the relative modulator enhancement is substantial). Trend lines of modest-response variants were again found to be very similar between CFBE and FRT cell lines (Figure 5D). Plotting of all variants studied revealed robust correlation between forskolin-stimulated CFTR function and iva/lum-enhanced CFTR function, illustrating that this relationship is independent of cell type and pathologic mechanism for each variant (Figure 5D).

To formally test whether iva/lum produces higher responses than either compound alone, we performed a detailed analysis of the 45 variants expressed in CFBE cells. Iva/lum combination treatment generated an equal or better response than either ivacaftor or lumacaftor for 30 of 33 variants that demonstrated modest responses to iva/lum (Figure 6A). The variants that did not demonstrate significantly better function with iva/lum were p.His139Arg (H139R), R334W, and p.Asp513Gly (D513G), and these variants also did not demonstrate a significant response to either ivacaftor or lumacaftor. Five rare variants were found to have an intermediate or high response to ivacaftor; for these 5 variants, iva/lum combination treatment was greater or equal to either ivacaftor or lumacaftor treatment (Figure 6B). Six variants were intermediate- or high-response variants to lumacaftor treatment; for these 6 variants, iva/lum combination treatment was greater or equal to either ivacaftor or lumacaftor treatment (Figure 6C). Notably, the iva/lum combination was never worse than ivacaftor or lumacaftor alone. Out of the 45 rare variants evaluated, the response to iva/lum was statistically significantly higher than forskolin-stimulated function for all variants except for H139R, R334W, and D513G. 

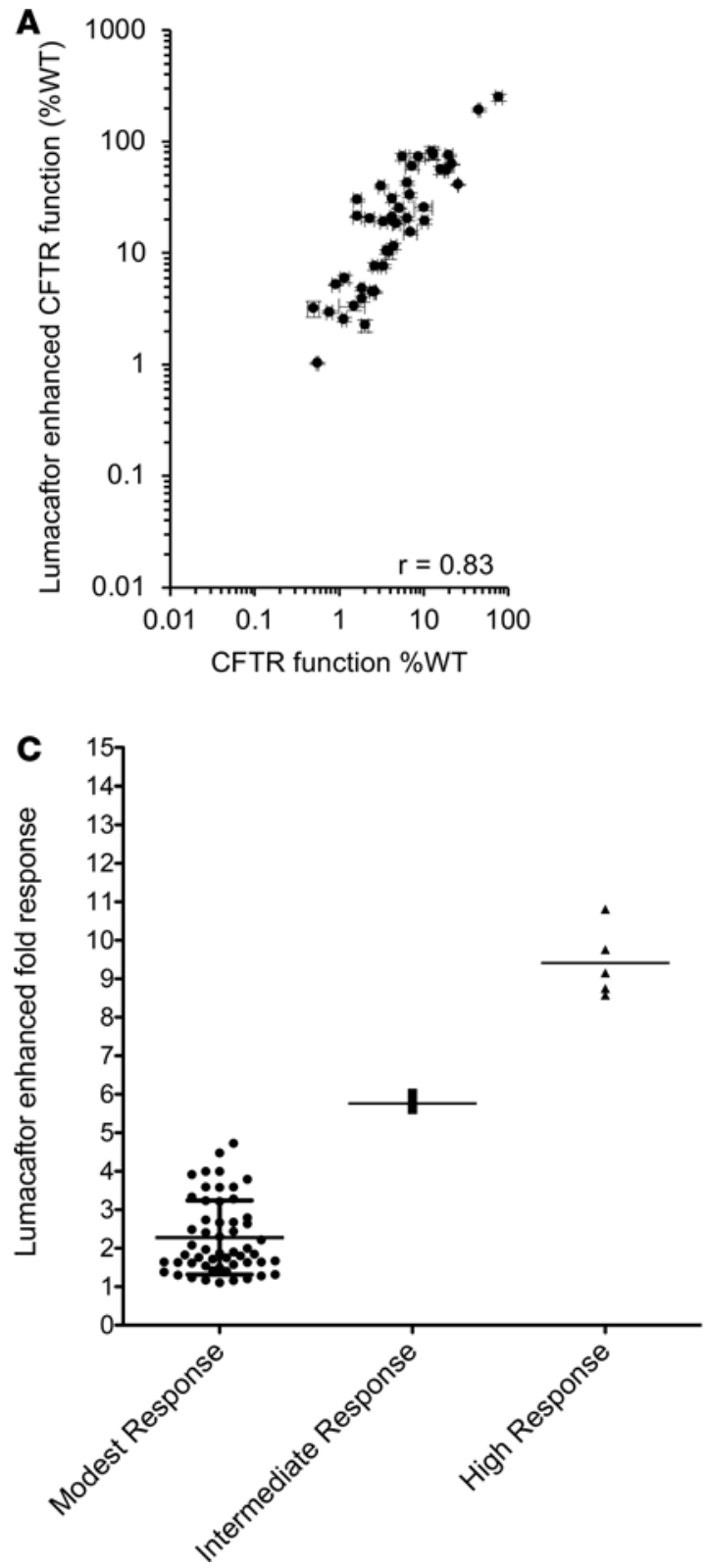
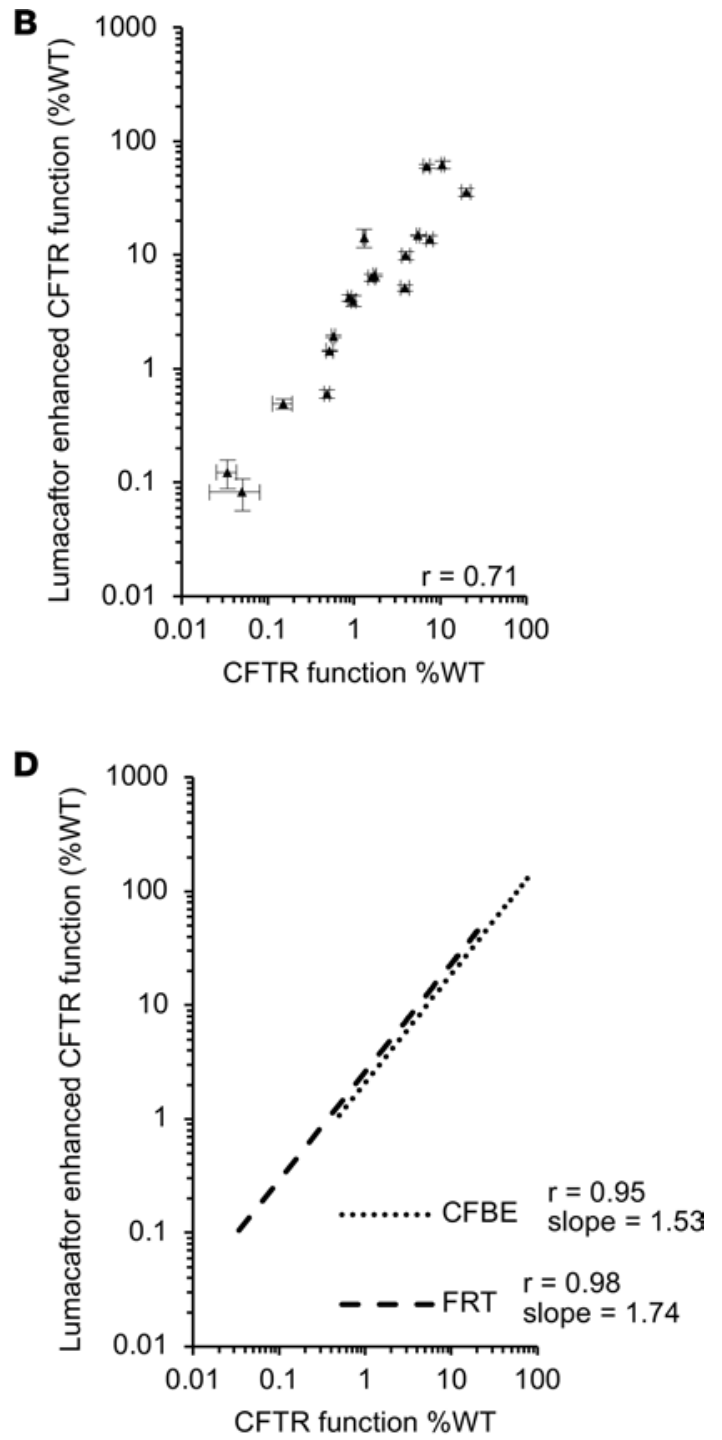

Figure 3. Lumacaftor response correlates with residual function. (A) Forskolin (10 $\mu \mathrm{M})$ stimulated CFTR function of 45 missense variants expressed in CF bronchial epithelial (CFBE) cells treated for 24 hours with $6 \mu \mathrm{M}$ lumacaftor compared with residual forskolin-stimulated (10 $\mu \mathrm{M})$ CFTR function when incubated for 24 hours with an equal volume of DMSO. Each variant was measured $n \geq 3$ and plotted as mean \pm SEM on both axes. (B) Forskolin-stimulated (5 $\mu \mathrm{M}$ ) CFTR function of 18 missense variants expressed in Fisher rat thyroid (FRT) cells treated for 48 hours with $3 \mu \mathrm{M}$ lumacaftor compared with residual forskolin-stimulated $(5 \mu \mathrm{M})$ CFTR function when incubated for 48 hours with an equal volume of DMSO. Each variant was measured $n \geq 3$ and plotted as mean \pm SEM on both axes. (C) Separation of variants based on their fold response to lumacaftor. Response of cell lines expressing G91R, E92K, L138ins, $\mathrm{L} 145 \mathrm{H}$, and L206W were designated as outliers by demonstrating fold response greater than $2 \mathrm{SD}$ beyond the mean fold response of all variants studied in CFBE and FRT cells, and they are labeled as high-response variants. Intermediate-response variants were those that remained outliers when high-response variants were removed from the comparison. All remaining variants were classified as modest response. Lines through data points represent the mean value $\pm 1 \mathrm{SD}$ for modest-response variants and mean of intermediate- and high-response variants. (D) Comparison of best fit functions for variants expressed in CFBE and FRT cells, which demonstrated modest response to lumacaftor. Correlation ( $r$ ) values calculated using Pearson linear correlation.

Although the trends for modest-response variants are the results that apply to the majority of variants and individuals with CF, the responses of the intermediate- and high-response variants also warrant evaluation. We compared the regression lines for modest-, intermediate-, and high-response variants for ivacaftor, lumacaftor, and iva/lum combination (Figure 7A). This analysis demonstrated that each response group yielded a steeper slope of the regression for all 3 treatments. These steeper slopes suggest that higher-response variants were distinguished by more effective modulator responses and were not the simple top end 
A

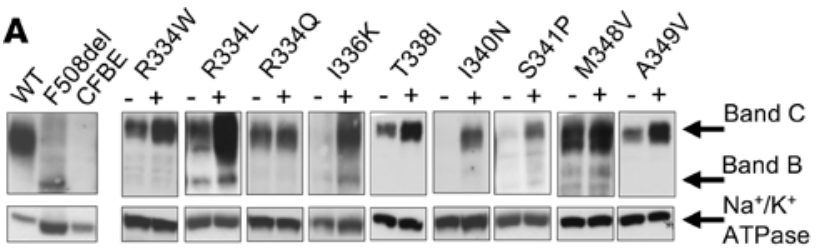

$-24 \mathrm{~h}$ DMSO

$+24 \mathrm{~h}$ Lumacaftor

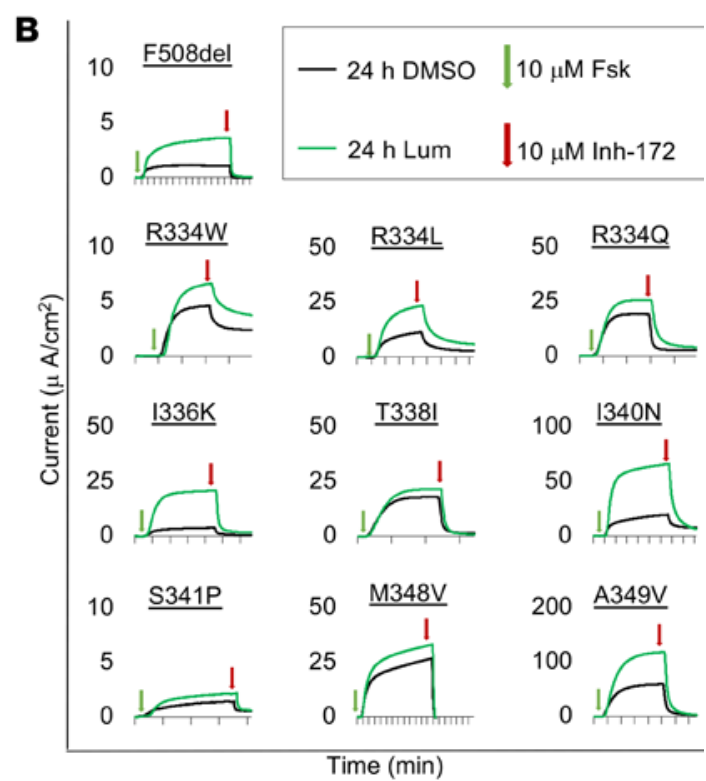

C

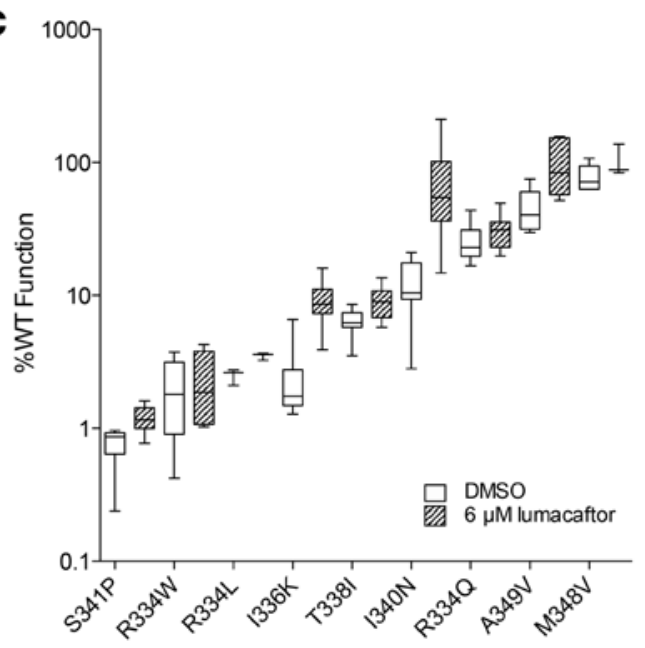

E

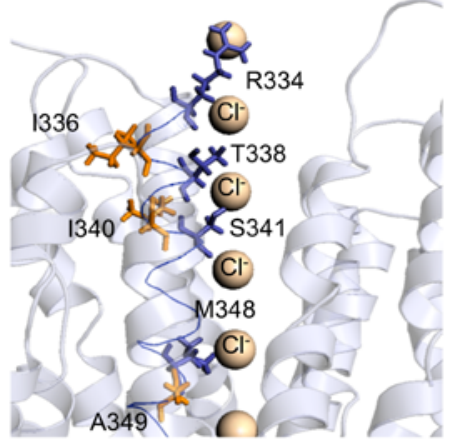

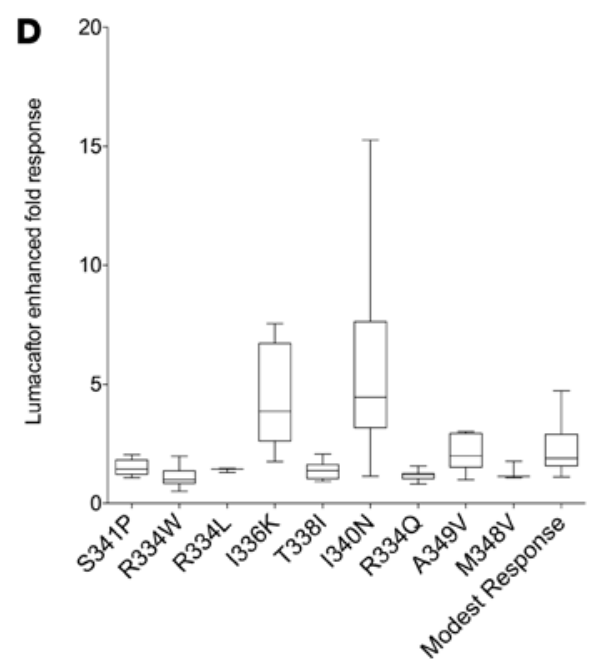

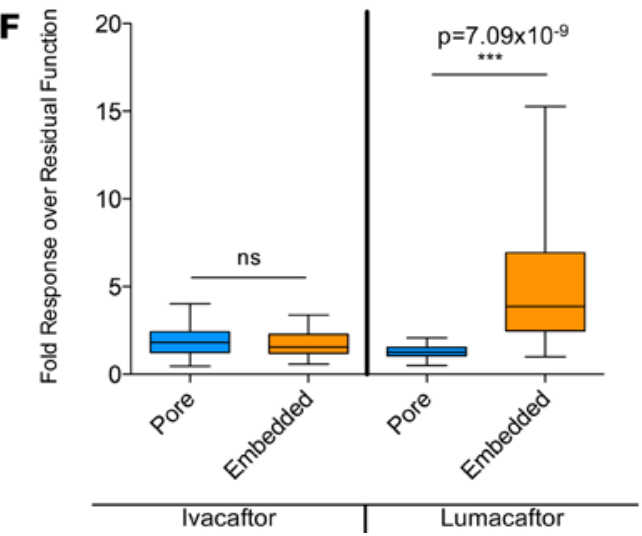

Figure 4. Variants located in sixth transmembrane domain (TM6) with exceptional response to lumacaftor corresponds to embedded side chain orientation within channel pore. (A) Western blots of whole cell lysates from CF bronchial epithelial (CFBE) cell lines stably expressing TM6 variants show that lumacaftor increases the quantity of mature CFTR protein for all TM6 variants. Data are representative of $n \geq 3$ for each variant. (B) $I_{s c}$ tracings of TM6 variants reveal that a subset of TM6 variants have an increased response to lumacaftor (lum) recorded as area corrected current ( $\mu \mathrm{A}$ / $\mathrm{cm}^{2}$ ), over time, measured in minutes represented by tick marks in 1-minute intervals. Data are representative of $n \geq 3$ for each variant. (C) Lumacaftor response of TM6 variants following 24-hour treatment with $6 \mu \mathrm{M}$ lumacaftor or an equal volume of DMSO. Box plots divide the data by quartile, with the median value indicated by a horizontal line within the box and whiskers extended to minimum and maximum values. (D) Lumacaftor response for each TM6 variant calculated as fold response over residual function compared with all modest-response variants identified in CFBE cells. Box plots divide the data by quartile, with the median value indicated by a horizontal line within the box and whiskers extended to minimum and 
maximum values. (E) Predicted orientation of TM6 residues within the CFTR conductance pore when in the open conformation (32). (F) Lumacaftor response relative to residual function for TM6 variants when grouped based on predicted orientation within the pore. Box plots divide the data by quartile, with the median value indicated by a horizontal line within the box and whiskers extended to minimum and maximum values.

of a normal distribution; we believe that the regressions would be parallel but elevated if the latter were the case. When we compared across each tier of response (modest, intermediate, and high) we found that the iva/lum combination generated the steepest regression for all 3 response levels (Figure 7B). Taken together, these results suggest that high-response variants are mechanistically distinct from modest-response variants and that iva/lum combination therapy is the best option for all variants, regardless of whether they are modest- or high-response variants.

\section{Discussion}

The CFTR modulators ivacaftor and lumacaftor have been approved for treatment of individuals with CF who carry a select set of variants based on the specific mechanistic defects of those variants. However, clinical studies of individuals with variants for which aberrant channel gating or folding is not the sole molecular defect, such as p.Pro67Leu (P67L) (50), p.Arg117His (R117H) (8), and other residual function variants (15, 51), have all shown clinical benefit from ivacaftor in a mechanism-independent manner. Using a wide range of variants expressed in 2 cell types, we show that residual CFTR function and drug response are highly correlated. Furthermore, we demonstrate that almost all tested variants generated higher currents in response to ivacaftor combined with lumacaftor than when treated with either compound alone. Following the common CF-causing variant F508del (52), missense variants represent the largest category of remaining variants associated with $\mathrm{CF}$ (3). Although the number of missense variants studied here encompass a small fraction of all missense variants reported in CFTR (57 of >800), they represent a significant portion of CF-causing alleles in the $\mathrm{CF}$ population. In total, the missense variants evaluated by this study represent $10 \%$ of all alleles reported to CFTR2 and 13,062 of 15,082 individuals (87\%) with at least 1 missense variant. Of added significance to the CF community moving forward, our results predict that greater clinical benefit would likely be derived from combinatorial therapy for most individuals with CF who carry missense variants and are given modulator compounds. The utility of this approach was recently demonstrated by a clinical trial employing modulator combinations (ivacaftor and tezacaftor) for individuals with residual CFTR function (15).

Heterologous expression of CFTR mutants in FRT cells has been used extensively to test modulator response, particularly to ivacaftor $(5,10,18,19,47)$. Based on the clinical efficacy and safety of ivacaftor, the FDA elected to accept results of CFTR testing in FRT cells as evidence to allow expansion of ivacaftor use without requiring clinical trials (20). This decision was reached based on the strong correlation between in vitro studies using FRT cells and clinical trials. Importantly, the results of those studies agreed, despite using a nonhuman cell line expressing a single allele of CFTR. This forward-thinking approach provides a path to treat every individual with CF who carries $C F T R$ variants that are sufficiently responsive to modulators, and it provides a mechanism to address barriers of formal clinical testing for $C F$ individuals with ultra-rare or even private mutations. However, the possibility remains that certain variants or certain modulators will require CFTR to function in its native human airway cellular context. To this end, we tested variants in CFBE cells and compared our results with the FRT model that has been well established as a system for studying CFTR modulators. The CFBE and FRT cell lines used here expressed CFTR in a controlled manner by stable expression from a single locus that guarded against artefactual interpretation of variants due to overexpression of the allele. For example, the finding of N1303K response to ivacaftor, if taken on its own, could be suggestive of clinical benefit, but the function in the expression-controlled FRT cell line demonstrates that the response is quite low and less likely to reach levels sufficient for clinical benefit. mRNA levels of CFTR expressed in CFBE cells were also determined to be within normal physiological limits of native human tissues by RNA sequencing (RNA-seq) studies. Despite their differences, we believe that our efforts to maintain controlled expression allowed CFBE and FRT cells to generate highly consistent results across a wide range of variants. The slopes and intercepts of correlation between residual function and modulator responses were remarkably similar, suggesting that the observed relationships were highly likely to be due to CFTR rather than cell-specific factors.

Ivacaftor increased chloride currents of G551D-CFTR by almost 7-fold in both cell lines, which is significantly less than was observed in previous studies in FRT cells $(18,19)$, but it is more consistent with studies performed in primary cells (5) and other cellular contexts $(6,7)$. The rare variants S1159F and S1159P, located 

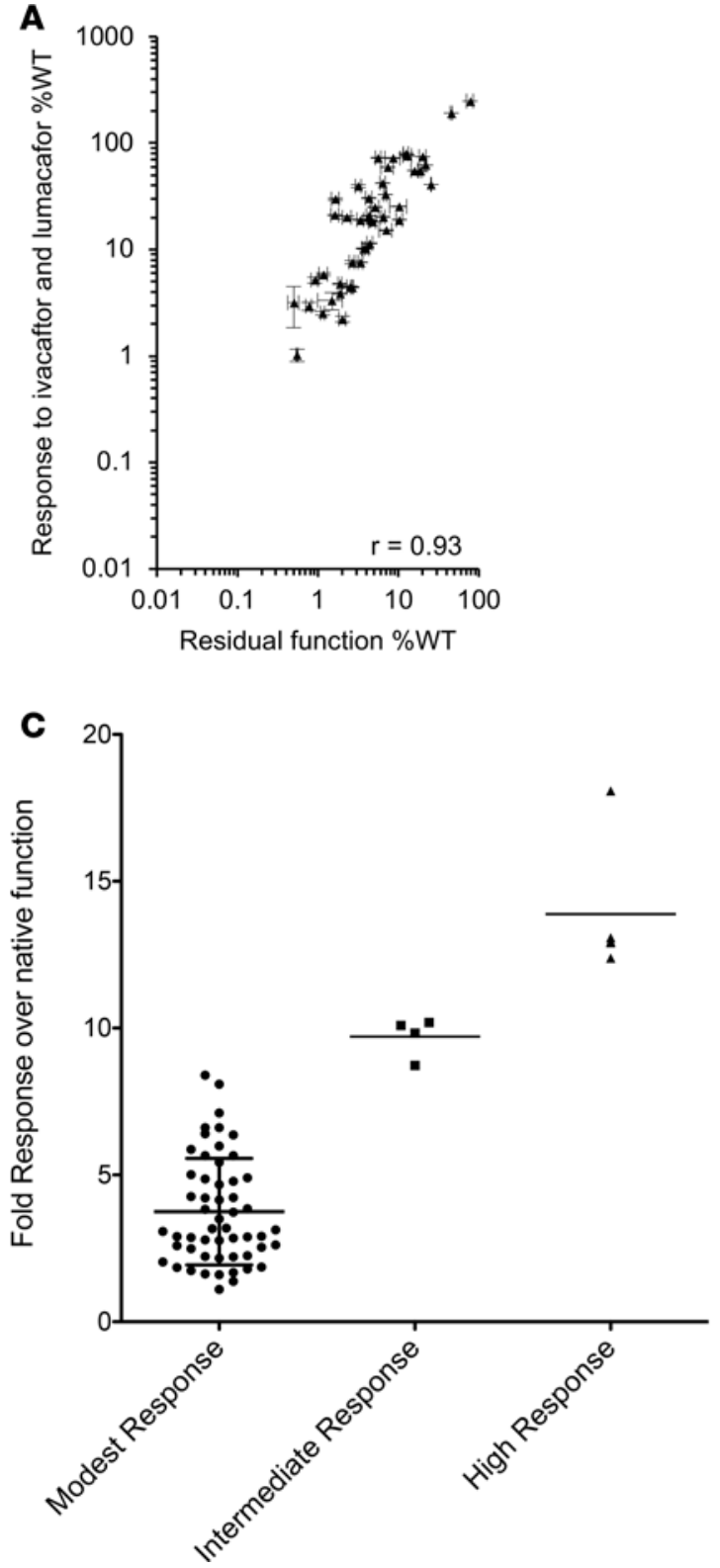

B
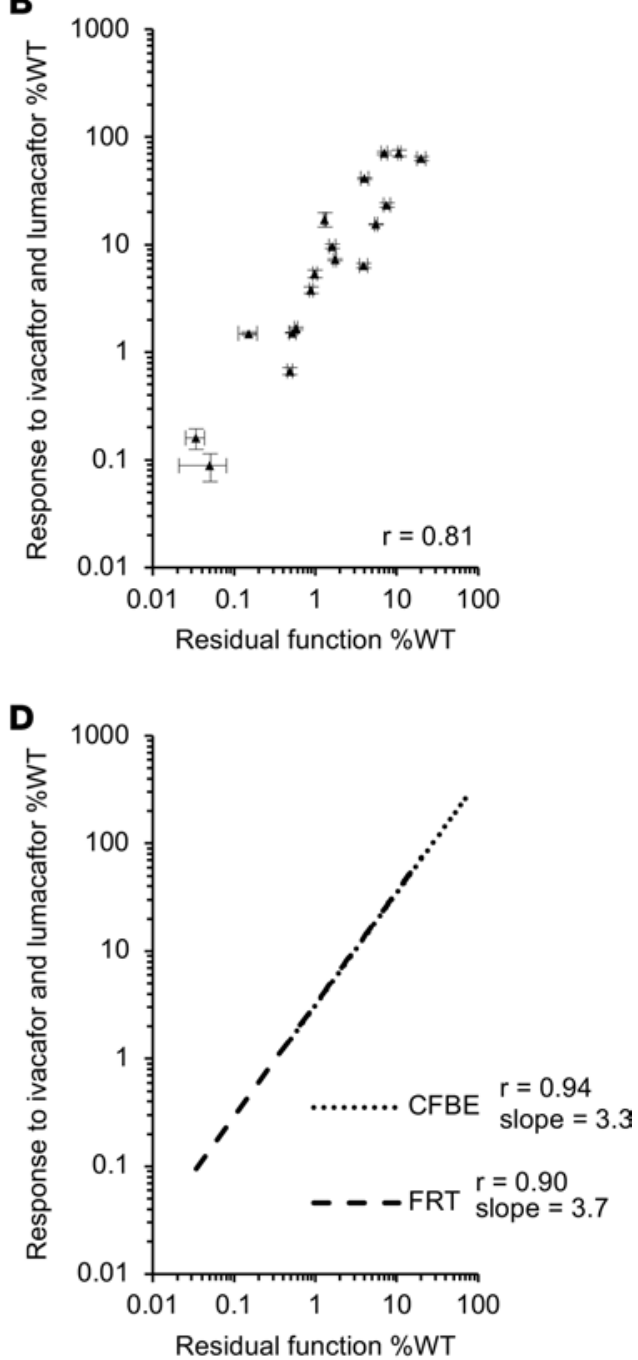

Figure 5. Ivacaftor/lumacaftor (iva/lum) response correlates with residual function. (A) Ivacaftor $(10 \mu \mathrm{M})$ enhanced CFTR function of 45 missense variants expressed in CFBE cells following for 24-hour incubation with $6 \mu \mathrm{M}$ lumacaftor compared with residual forskolin-stimulated (10 $\mu$ M) CFTR function when incubated for 24 hours with DMSO. Each variant was measured $n \geq 3$ and plotted as mean \pm SEM. (B) Ivacaftor (5 $\mu$ M) enhanced CFTR function of 18 missense variants expressed in FRT cells following 24-hour incubation with $3 \mu \mathrm{M}$ lumacaftor compared with residual forskolin-stimulated ( $5 \mu \mathrm{M}$ ) CFTR function when incubated for 24 hours with DMSO. Each variant was measured $n \geq 3$ and plotted as mean \pm SEM. (C) Separation of variants based on their fold response to lumacaftor. Response of cell lines expressing G91R, E92K, L138ins, L145H, and G551D (CFBE) were designated as outliers by demonstrating a fold response greater than 2 SD beyond the mean fold response of all variants studied in CFBE and FRT cells, and they are labeled as high-response variants. Intermediate-response variants were those that remained outliers when high-response variants were removed from the comparison. All remaining variants were classified as modest response. Lines through data points represent the mean value $\pm 1 \mathrm{SD}$ for modest-response variants and mean of intermediate- and high-response variants. (D) Comparison of best fit functions for variants expressed in CFBE and FRT cells that demonstrated modest response to ivacaftor/ lumacaftor combination treatment. Correlation $(r$ ) values calculated using Pearson linear correlation.

prior to the second nucleotide-binding domain (NBD2) (53), also exhibited a high response to ivacaftor. Even after removing high-response variants from the analysis, a second set of variants demonstrated a response to ivacaftor that was significantly elevated compared with the remaining variants. The high degree of correlation between residual function and modulator response for modest-response variants $(r=0.94)$ suggests that baseline function might predict ivacaftor response for all variants and not just the ones tested here. Although absolute response to modulators may not be sufficient for very low-function variants to demonstrate significant 
A

Modest response variants

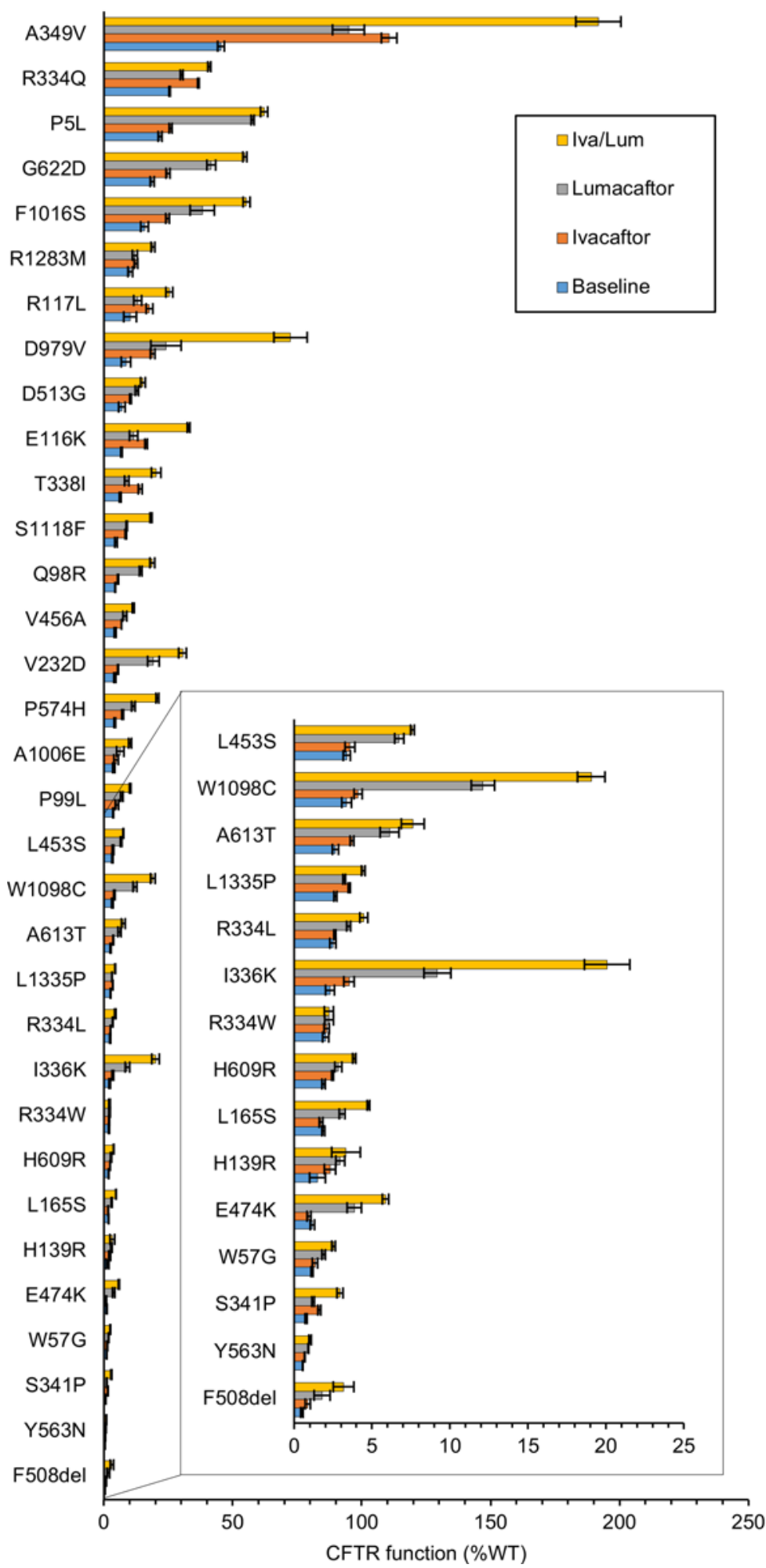

B Ivacaftor responsive variants

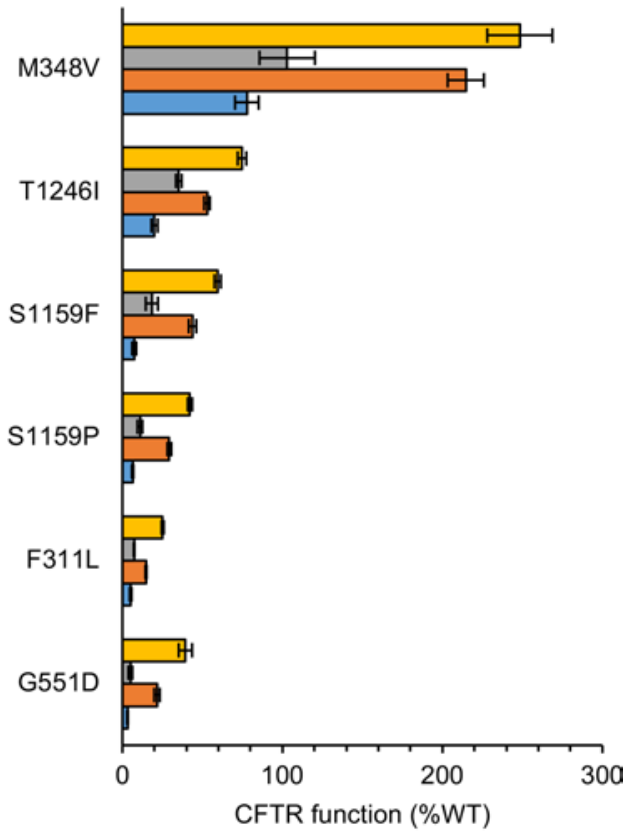

C Lumacaftor responsive variants

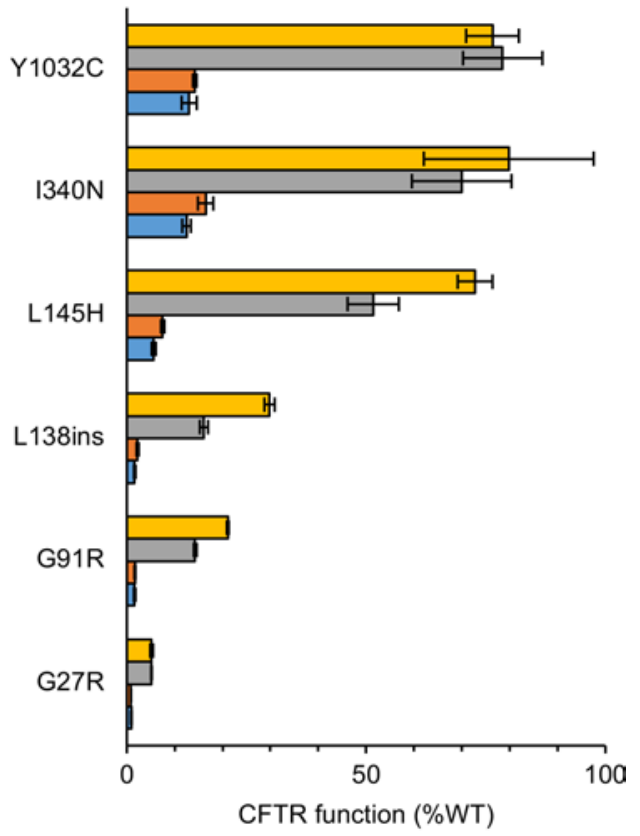

Figure 6. Summary of response of missense variants to CFTR modulators. (A) CFTR function for variants that were modest-response variants for ivacaftor and lumacaftor when treated with forskolin (baseline), ivacaftor, lumacaftor, or iva/lum combination. Error bars represent \pm SEM.

(B) Response to ivacaftor, lumacaftor, or ivacaftor/lumacaftor combination for variants designated as intermediate- or high-response to ivacaftor.

(C) Response to ivacaftor, lumacaftor, or iva/lum combination for variants designated as intermediate or high response to lumacaftor. 
A Modest Response _ - . Intermediate Response $\quad \cdots . . .$. High Response $\quad \mathrm{n}$ - \# of variants
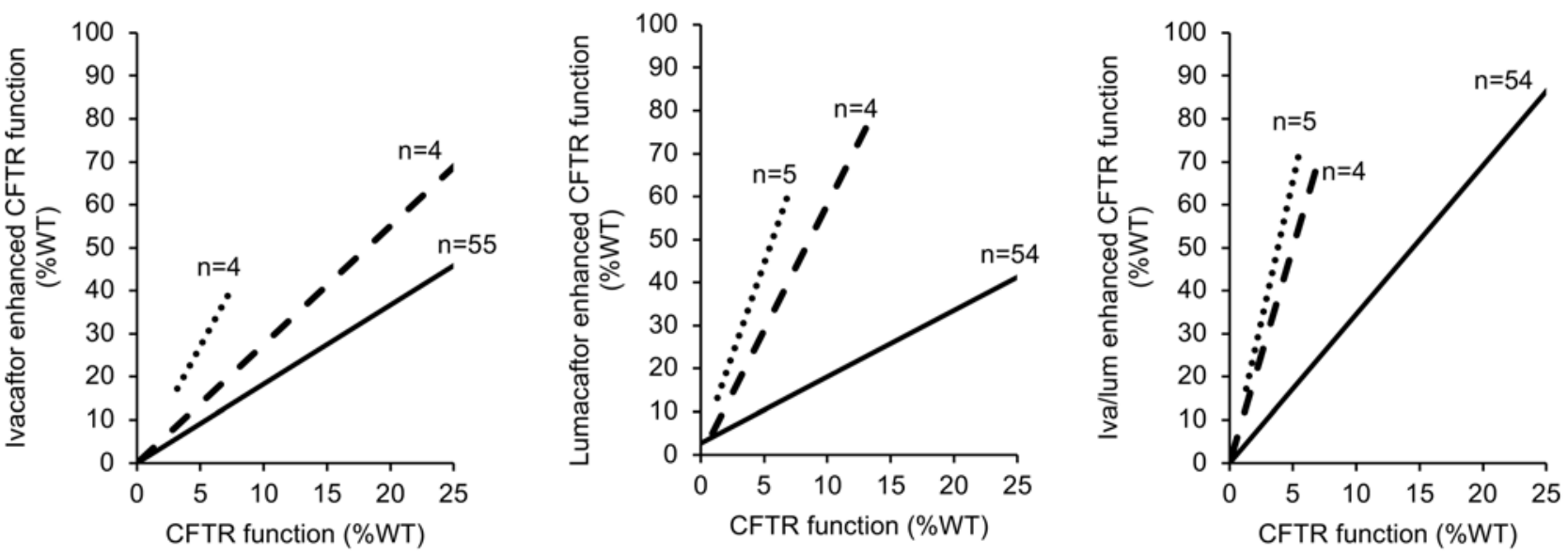

B

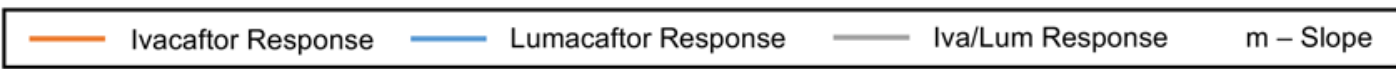
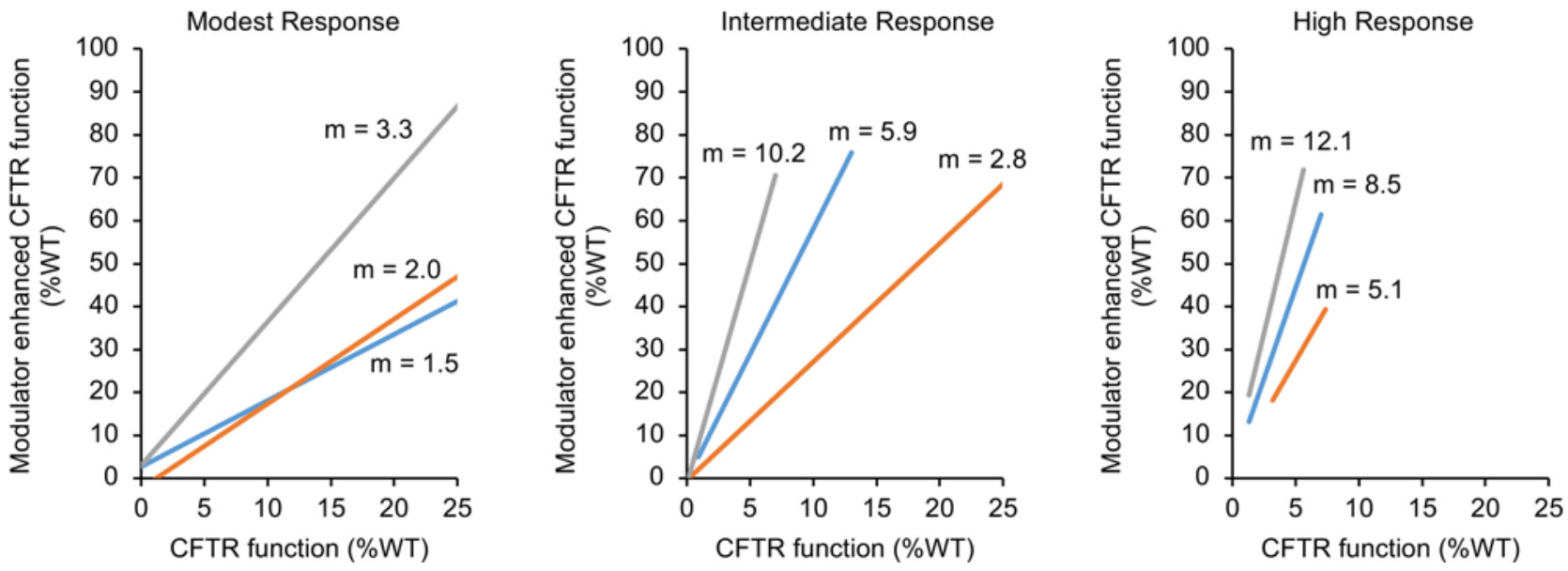

Figure 7. Combination therapy yields larger response than monotherapy across all response tiers. (A) Comparison of response trends for ivacaftor (left), lumacaftor (middle), and ivacaftor/lumacaftor combination (right) of all variants studied in CF bronchial epithelial (CFBE) and Fisher rat thyroid (FRT) cell lines. (B) Comparison of response trends for all 3 treatment strategies for modest-response variants (left), intermediate-response variants (middle), and high-response variants (right).

clinical benefit (i.e., crossing a 10\% WT function disease threshold), evaluation by fold change could inform mechanism of action. Such mechanisms may be distinct from potentiator compounds that promote NBD dimerization (54) and guide rationale design of more effective potentiators. In addition, differentiating variants that have exceptional responses will be important to establish accurate expectations for clinical outcomes.

The effect of lumacaftor on CFTR-bearing missense variants has been less extensively studied than for ivacaftor, and the present study, to our knowledge, reports the largest collection of variants tested for their response to lumacaftor. Lumacaftor was designed to target the common F508del variant, and we observed increased CFTR function in cell lines expressing F508del when treated with this compound. Although we were unable to fully recapitulate the magnitude of effect previously published (10), we believe our findings of a 4 -fold increase in function from $0.5 \%-2 \%$ WT function are concordant with results from clinical studies demonstrating the ability of lumacaftor to reduce sweat chloride in a dose-dependent manner up to a reduction of $6.6 \mathrm{mmol} / 1$ while being unable to significantly improve lung function (12) or other biomarkers of CFTR function (55). Correlation between residual CFTR function and lumacaftor response had previously 
been observed in studies of primary nasal epithelial cells bearing a small series of missense variants (56). Furthermore, in vitro and ex vivo studies of missense variants demonstrate increased CFTR function following treatment with lumacaftor $(46,57)$. Thus, it is reasonable to expect that the observations in the CFBE and FRT cell lines will be relevant in vivo. We also identified missense variants that respond significantly better to lumacaftor than F508del (i.e., greater than 2 SD above the mean). These results suggest that individuals carrying certain variants might respond better to lumacaftor than those who carry F508del. Although intermediate and high lumacaftor responses applied only to 9 variants, lumacaftor increased the CFTR function of most variants tested, regardless of the mechanism underlying their CFTR dysfunction. Thus, many individuals with CF who carry variants that allow protein production may also benefit from lumacaftor treatment. Given that tezacaftor is functionally similar to lumacaftor (58), we predict that the same benefit would be obtained when using this CFTR corrector, similar to what has been observed in studies of primary cells (56).

The location of the residues that confer high lumacaftor responses could inform the future development of correctors. Of the 6 variants that demonstrated robust responses to lumacaftor, 4 are located in the TMDs (E92K, L206W, I336K, and I340N), while the 2 variants with the highest responses are located in the ICLs: L138ins and L145H near the first ICL (ICL1) and Y1032C near the interface of TM10 and ICL4. Recent evidence indicates that lumacaftor promotes interactions between ICL1 and NBD1 (59), as well as between ICL4 and NBD1 (48). Thus, the significant increases in current above baseline exhibited by CFTR bearing L138ins and $\mathrm{L} 145 \mathrm{H}$ upon treatment with lumacaftor might be a consequence of their locations. As lumacaftor is thought to rescue CFTR function by improving protein folding and, hence, structure, we mapped the location of all TM6 variants studied here onto models (32) and structures (36) of the CFTR protein. I336K and I340N are found in TM6 at residues where cryo-EM structures $(36,37)$, homology modeling (32), and experimental evidence predicts that they are embedded (42, 43, 49), whereas all other variants, except for p.Ala349Val (A349V), are predicted to be oriented into the open channel pore. Although the lumacaftor response of $\mathrm{A} 349 \mathrm{~V}$ was not found to be significantly different from all other missense variants, it was significantly higher than other TM6 variants $(P<0.05)$. These results indicate that missense variants that alter ion conductance by disruption of the structure of the pore are mechanistically distinct from those that more directly impede ion flow. Amelioration of local folding defects imposed on the channel pore by variants such as $\mathrm{I} 336 \mathrm{~K}$ and $\mathrm{I} 340 \mathrm{~N}$ might underlie the prominent response of these variants to lumacaftor.

Our extensive evaluation of responses of variants expressed in CFBE cells confirmed that the majority of variants (42 of 45) demonstrated increased CFTR function when treated with iva/lum. Moreover, 26 of 45 variants demonstrated greater responses to the combination than to ivacaftor or lumacaftor alone, while 16 others showed an equal response to ivacaftor or lumacaftor that was higher than baseline function, and the combination was not found to be less effective than either individual modulator for any variant tested. Although some studies have observed antagonism between ivacaftor and lumacaftor for some genotypes $(57,60)$, we saw no evidence of antagonistic effects between these compounds in either CFBE or FRT systems. The regression functions suggest that a minimum ( $2 \% \mathrm{WT})$ amount of residual function is required for ivacaftor action, likely due to the requirement of well-folded and processed protein localized to the cell surface, while lumacaftor has no such requirement. The regression function for iva/lum combination treatment removes the minimum function requirement for response, suggesting that lumacaftor action results in ivacaftor response. It is known that F508del results in multiple molecular defects that require correction $(61,62)$; therefore, combination therapy should be expected to be the most effective treatment for F508del (63) and other variants that have folding and activity defects. Combination therapy was also effective for variants with high responses to ivacaftor or lumacaftor, including G551D, indicating that individuals bearing these variants could additionally benefit from combinatorial therapy.

Of significant note, the modest-response variants treated with the iva/lum combination crossed 10\% WT function for variants with $3 \%$ baseline function. The threshold of $3 \%$ residual WT function as a differentiator of predicted alleviation of lung disease for combinatorial modulator therapy is important, as this level of CFTR activity also coincides with an approximate threshold for exocrine pancreatic sufficiency (64). Pancreatic sufficiency of an individual with CF can be determined independently of genetic testing, thus potentially removing hurdles to interpretation of genetic variants - including incomplete genotyping, variants of uncertain significance, errors in genetic test reports, and extreme rarity of missense variants, among other considerations. Given the high correlation between baseline function and modulator response for modest-response variants $(r=0.94)$, residual function of an individual may be a reasonable predictor of drug response, which has been postulated previously based on studies of primary cells of a limited number of genotypes (56). 
The exceptions to the modest responses to ivacaftor and lumacaftor are significant for individuals carrying those variants, as well as for informing mechanism and design of future CFTR modulators. Individuals who carry these highly responsive variants may be able to achieve significant levels of CFTR function and improved clinical outcomes, even if their CFTR function without treatment is poor. Furthermore, understanding the mechanism underlying the strong response may inform development of more efficient versions of these modulators, which can then confer larger responses to individuals carrying modest-response variants, as well. While these high-response variants might lead to significant improvements in clinical outcome, it is important to note different tiers of response when discussing therapeutic outcomes with patients. The specific level of function required to escape lung disease will vary to some degree among individuals due to modifier genes, environmental factors, and/or stochastic factors unique to each individual, as noted in studies of twins and siblings (65). The level of CFTR function required to demonstrate clinical benefit may in some cases be as low as $5 \%$, as implied by RNA studies (66), or possibly as high as $25 \%$, which was necessary to restore full ciliary function (67). Of note, the conservative threshold of $10 \% \mathrm{WT}$ function is higher than what was achieved for combinatorial treatment of F508del in both FRT and CFBE systems. This is important, since iva/lum has achieved clinical benefit and FDA approval for individuals who carry 2 copies of F508del (13). If the response of mutant CFTR to newer derivatives and novel classes of modulators continues to correlate with residual function, it is possible that therapeutic response might be achieved in individuals with very minimal residual function.

\section{Methods}

\section{Study design}

Rare variants from the CFTR2 database that had moderate sweat chloride (40-90 mM) were selected for study in CFBE cells. The set of moderate sweat variants were ranked in priority based on frequency within the CF population. Variants that had $<10 \%$ WT function were selected to evaluate drug responses. Several of the first cell lines made were also tested for drug response even if their residual function was greater than 10\% WT (D979V, Y1032C, G622D, and P5L). Missense variants from TM6 were based on previous studies $(18,31)(\mathrm{R} 334 \mathrm{~W}, \mathrm{I} 336 \mathrm{~K}, \mathrm{~T} 338 \mathrm{I}$, and S341P) or naturally occurring variants at positions of interest (R334L, R334Q, I340N, M348V, and A349V). All TM6 variants were tested for drug response, regardless of residual function. In total, variants were selected across a range of clinical and molecular phenotypes to identify trends that might be useful for predicting the drug response of novel missense variants identified in individuals without the need for in vitro testing. Functional testing was measured with a minimum of $n=3$ for each cell line tested for each drug condition, as well as a minimum of $n=3$ for measurement of CFTR expression by quantitative PCR (qPCR). Drug doses were assigned based on previous studies $(5,10,18,19$, 21) and compared with equivalent treatment with the drug vehicle (DMSO in all cases).

\section{Generation of variant plasmids}

CFBE cells. CFTR expression plasmids containing WT CFTR cDNA sequence used for generation of CFBE cell lines were provided by J. Rommens (University of Toronto, Toronto, Ontario, Canada). Plasmid was modified to utilize the human EF1a promoter (21), to revert the V1475M polymorphism (21), and to include the V470M polymorphism for every variant known to carry it in cis. This plasmid was used as a template for generation of variant CFTR alleles by site-directed mutagenesis. The complete CFTR cDNA was sequenced to guard against secondary mutations. Plasmids were confirmed to be competent for transfection by transient transfection of HEK293 cells and subsequent Western blotting to evaluate production of protein (Supplemental Methods).

FRT cells. pcDNA5/FRT expression plasmid used for generation of FRT cell lines was purchased from Thermo Fisher Scientific. CFTR cDNAs encoding variants were cloned in between NotI and XhoI restriction sites of the pcDNA5/FRT expression vector. All variants encoded the common V470M polymorphism except 3 (G85E, R347P, and M1101K), to reflect haplotypes commonly associated with these alleles.

\section{Generation of stable cell lines}

CFBE cells provided were by D. Gruenert (UCSF, San Francisco, California, USA). Cell lines were modified to include $\mathrm{Flp}$ recombinase integration site and mutate the large $\mathrm{T}$ antigen binding site, as previously described (21). Cells were transfected with CFTR variant plasmids in the presence of the pOG44 recombinase plasmid. Cells were grown under hygromycin selection $(100 \mu \mathrm{g} / \mathrm{ml})$, and individual clones were 
picked using cloning cylinders and were grown/expanded separately. CFTR expression level was confirmed by qPCR (Supplemental Methods).

Isogenic FRT cells were a gift from M. Welsh (University of Iowa, Iowa City, Iowa, USA).

FRT cell lines were generated using the Flp-In system (Thermo Fisher Scientific) following previously published methods $(18,26)$. Multiple clones of FRT cells expressing each variant were isolated, and CFTR mRNA levels were compared with an established WT CFTR line obtained by the same protocol. Cell lines with mRNA levels comparable to WT (within $0.5-1.5$ fold) were selected for further study.

\section{Measurement of CFTR function}

CFBE. CFTR-mediated transepithelial $\mathrm{Cl}^{-}$currents were recorded using a $\left[\mathrm{Cl}^{-}\right]$gradient to magnify current size without permeabilizing the basolateral membrane as previously described $(21,46)$. Cells $\left(1 \times 10^{5}\right)$ were plated on Snapwell filters (Corning) and cultured for 6 days while submerged to a minimum transepithelial resistance $\left(\mathrm{R}_{\mathrm{t}}\right)$ of $200 \Omega \times \mathrm{cm}^{2}$, while $\mathrm{I}_{\mathrm{sc}}$ measurements were performed with EasyMount Ussing chambers (Physiologic Instruments) using a chloride concentration gradient with high chloride buffer in the basolateral chamber and low chloride buffer in the apical chamber (Supplemental Methods). Air was gently bubbled into the buffers to promote circulation. After cancelling voltage offsets, transepithelial voltage was clamped (referenced to the basolateral solution) at $0 \mathrm{mV}$ and $\mathrm{I}_{\mathrm{sc}}$ recorded continuously. Once currents had stabilized, forskolin (Selleckchem) was administered to the basolateral chamber at a final concentration of $10 \mu \mathrm{M}$. Once stabilized at the maximal forskolin-stimulated current level, the inhibitor Inh-172 (Selleckchem) was administered to the apical chamber at a final concentration of $10 \mu \mathrm{M}$. The contribution of CFTR function to overall current was measured as the decline in current following administration of Inh-172 $\left(\Delta \mathrm{I}_{\mathrm{sc}}\right)$. Assessment of the functional consequence of CFTR missense variants was performed by normalizing the $\Delta \mathrm{I}_{\mathrm{sc}}$ based on mRNA quantity to quantify percent-WT function as described previously (22) (Supplemental Methods).

FRT. FRT cells $\left(1.5 \times 10^{5}\right)$ were seeded and cultured on Transwell permeable supports (Corning) for 5 days to form well-polarized monolayers with a minimum $\mathrm{R}_{t}$ of $400 \Omega \times \mathrm{cm}^{2}$. $\mathrm{I}_{\mathrm{sc}}$ was evaluated in the presence of basolateral to apical chloride concentration gradient (Supplemental Methods) with an EasyMount Ussing Chamber System (Physiologic Instruments) and Acquire and Analyze software. Once current stabilized, $100 \mu \mathrm{M}$ amiloride was applied to both the apical and basolateral sides to inhibit the epithelial sodium channel ENaC. To activate the CFTR-specific chloride current, $5 \mu \mathrm{M}$ forskolin (MilliporeSigma) was added to both sides. Inh-172 (10 $\mu \mathrm{M}$; MilliporeSigma) was applied to the apical side to inhibit CFTR-mediated current. The process for determining percent-WT function in FRT cells was similar to that previously reported (68) but used a narrow range of mRNA expression (Supplemental Methods).

\section{Western blots of stable cell lines}

Lysate (100 $\mu \mathrm{g}$ of total) was collected from CFBE cells. Blots were probed with anti-CFTR mouse monoclonal antibody 596, provided by Cystic Fibrosis Foundation Therapeutics (J. Riordan, University of North Carolina, Chapel Hill, North Carolina, USA) at a concentration of 1:1,000 and secondary anti-mouse antibody (GE Healthcare, catalog NA931V) at a concentration of 1:100,000. The sodium potassium $\left(\mathrm{Na}^{+} / \mathrm{K}^{+}\right)$ATPase was used as a loading control for all blots; primary antibody was rabbit monoclonal antibody (Abcam, catalog ab76020, clone EP1845Y) at a concentration of 1:50,000 and secondary anti-rabbit (GE Healthcare, cata$\log$ NA934V) antibody concentration was 1:200,000. All blots were imaged using ECL Prime reagent (GE Healthcare) and x-ray film.

\section{Potentiator testing}

CFBE cells were prepared for $I_{s c}$ measurements as described above. Following stabilization of the forskolin-stimulated current, ivacaftor (Selleckchem) was administered to the apical chamber at a final concentration of $10 \mu \mathrm{M}$. Potentiators provided by the CFFT CFTR Chemical Compound Program (R. Bridges, Rosalind Franklin University of Medicine and Science, North Chicago, Illinois, USA) were also administered into the apical chambers at concentrations corresponding to their published half-maximal effective concentration $\left(\mathrm{EC}_{50}\right.$ ) values (69-73). Inh-172 was applied to measure the CFTR portion of the current. The effects of potentiators were measured as the Inh-172-inhibited portion $\left(\Delta \mathrm{I}_{\text {sc }}\right)$ of the current when stimulated by forskolin and then enhanced by the potentiator compared with the $\Delta \mathrm{I}_{\mathrm{sc}}$ when stimulated by forskolin only. 
FRT cells were prepared for $\mathrm{I}_{\mathrm{sc}}$ measurements as described above. Following stabilization of the forskolin-stimulated current, $5 \mu \mathrm{M}$ ivacaftor (Selleckchem) was applied to the apical side only. Finally, $10 \mu \mathrm{M}$ inh172 (MilliporeSigma) was applied to the apical side to inhibit CFTR-mediated current. Ivacaftor-enhanced function was measured as the $\Delta \mathrm{I}_{\mathrm{sc}}$ of the ivacaftor-enhanced forskolin-activated current and compared with $\Delta \mathrm{I}_{\mathrm{sc}}$ when stimulated by forskolin only.

\section{Corrector testing}

CFBE cells were grown in the presence of corrector compound or equivalent volume of DMSO for 24 hours. Lumacaftor (Selleckchem) was administered at a final concentration of $6 \mu \mathrm{M}$, while corrector compounds provided by the CFFT CFTR Chemical Compound Program (R. Bridges, Rosalind Franklin University of Medicine and Science) were tested at concentrations corresponding to their $\mathrm{EC}_{50}$ values (74-79). The effects of correctors on CFTR function were evaluated by $\mathrm{I}_{\text {sc }}$ comparison of forskolin-stimulated CFTR currents of cells treated with each compound compared with the DMSO-treated negative control. To evaluate the effect of lumacaftor on protein processing, total protein lysates were collected from stable cells after 24-hour incubation with $3 \mu \mathrm{M}$ lumacaftor or an equivalent volume of DMSO, and Western blots were performed as described above.

FRT cells were prepared for $\mathrm{I}_{\mathrm{sc}}$ measurements as described above. Forty-eight hours prior to assay, cells were treated with $3 \mu \mathrm{M}$ lumacaftor (Selleckchem) or DMSO from both apical and basolateral surfaces of the epithelia. The effects of correctors on CFTR function were evaluated by $\mathrm{I}_{\mathrm{sc}}$ comparison of forskolin-stimulated CFTR currents of cells treated with each compound compared with the DMSO-treated negative control.

\section{Structural analysis}

Cryo-EM structures and homology models of the open conformation of CFTR $(32,36,37)$ were used to map the position and orientation of the residues studied here using Pymol software and publicly available Protein Data Bank (PDB) files (5W81,5UAK, 2ONJ, and 2HYD).

\section{Statistics}

Designation of modulator response outliers was performed by designating those responses with fold changes greater than 2 SD above the mean as high-response variants. High-response variants were removed from the data set, and outliers from the remaining data set (greater than 2 SD above the mean) were designated as intermediate-response variants; all other responses were designated as modest-response variants. Results are presented as mean values \pm SEM for scatter plots and bar plots. Box plots divide the data by quartile, with the median value indicated by a horizontal line within the box and whiskers extended to minimum and maximum values. Correlations were calculated by Pearson linear correlations. Statistical significance was calculated by Student's unpaired 2 tailed $t$ tests. Differences were considered statistically significant when $P<0.05$. Complete individual data sets for each cell line are available in Supplemental Table 1.

\section{Author contributions}

STH designed studies for CFBE cells, generated and analyzed data for CFBE cells, and wrote the manuscript. AR designed studies for FRT cells, generated and analyzed data for FRT cells, and reviewed the manuscript. MJP, EFD, AFM, TAE, ATJ, and ZL contributed to collection of data for CFBE cells. ZC performed all single-channel experiments. KSR provided information on missense variants found in the CF population. $\mathrm{JH}$ and EJS designed studies for FRT cells and reviewed the manuscript. DNS designed studies for single-channel experiments and reviewed the manuscript. GRC was responsible for overall study design, development of the CFBE cell expression system, critical review of all results, and writing and editing the manuscript.

\section{Acknowledgments}

We thank the CFTR2 project for variant data, R.J. Bridges and CFFT for panels of small molecule CFTR potentiators and correctors, J.R. Riordan and CFFT for anti-CFTR antibodies, P. Thomas and L. Millen for N1303K cDNA construct used for single-channel studies, C.A. Cotton for identification of the L145H variant, M.J. Welsh for isogenic FRT cell lines, and F. Van Goor and Vertex Pharmaceuticals for access to ivacaftor response data generated using FRT cells. This work was supported by the NIH (R01DK44003), CF Foundation (Cuttin13A1, Cuttin15XX0, and Cuttin16IO to GRC; SORSCH13XX0 and SORSCH14XX0 to EJS), and CFFT (SHEPPA14XX0) to DNS 
Address correspondence to: Garry Cutting, The Johns Hopkins Medical Institutions, 733 North Broadway, BRB 559, Baltimore, Maryland 21205, USA. Phone: 410.614.0211; Email: gcutting@jhmi.edu.

1. Riordan JR, et al. Identification of the cystic fibrosis gene: cloning and characterization of complementary DNA. Science. 1989;245(4922):1066-1073.

2. Gadsby DC, Vergani P, Csanády L. The ABC protein turned chloride channel whose failure causes cystic fibrosis. Nature. 2006;440(7083):477-483.

3. Cutting GR. Cystic fibrosis genetics: from molecular understanding to clinical application. Nat Rev Genet. 2015;16(1):45-56.

4. Oliver KE, Han ST, Sorscher EJ, Cutting GR. Transformative therapies for rare CFTR missense alleles. Curr Opin Pharmacol. 2017;34:76-82.

5. Van Goor F, et al. Rescue of CF airway epithelial cell function in vitro by a CFTR potentiator, VX-770. Proc Natl Acad Sci USA. 2009;106(44):18825-18830.

6. Eckford PD, Li C, Ramjeesingh M, Bear CE. Cystic fibrosis transmembrane conductance regulator (CFTR) potentiator VX-770 (ivacaftor) opens the defective channel gate of mutant CFTR in a phosphorylation-dependent but ATP-independent manner. J Biol Chem. 2012;287(44):36639-36649.

7. Jih KY, Hwang TC. Vx-770 potentiates CFTR function by promoting decoupling between the gating cycle and ATP hydrolysis cycle. Proc Natl Acad Sci USA. 2013;110(11):4404-4409.

8. Moss RB, et al. Efficacy and safety of ivacaftor in patients with cystic fibrosis who have an Arg117His-CFTR mutation: a double-blind, randomised controlled trial. Lancet Respir Med. 2015;3(7):524-533.

9. De Boeck K, et al. Efficacy and safety of ivacaftor in patients with cystic fibrosis and a non-G551D gating mutation. $J$ Cyst Fibros. 2014;13(6):674-680.

10. Van Goor F, et al. Correction of the F508del-CFTR protein processing defect in vitro by the investigational drug VX-809. Proc Natl Acad Sci USA. 2011;108(46):18843-18848.

11. Lukacs GL, Verkman AS. CFTR: folding, misfolding and correcting the $\Delta$ F508 conformational defect. Trends Mol Med. 2012;18(2):81-91.

12. Clancy JP, et al. Results of a phase IIa study of VX-809, an investigational CFTR corrector compound, in subjects with cystic fibrosis homozygous for the F508del-CFTR mutation. Thorax. 2012;67(1):12-18.

13. Rehman A, Baloch NU, Janahi IA. Lumacaftor-Ivacaftor in Patients with Cystic Fibrosis Homozygous for Phe508del CFTR N Engl J Med. 2015;373(18):1783.

14. Taylor-Cousar JL, et al. Tezacaftor-Ivacaftor in Patients with Cystic Fibrosis Homozygous for Phe508del. N Engl J Med. 2017;377(21):2013-2023.

15. Rowe SM, et al. Tezacaftor-Ivacaftor in Residual-Function Heterozygotes with Cystic Fibrosis. N Engl J Med. 2017;377(21):2024-2035.

16. Sheppard DN, Carson MR, Ostedgaard LS, Denning GM, Welsh MJ. Expression of cystic fibrosis transmembrane conductance regulator in a model epithelium. Am J Physiol. 1994;266(4 Pt 1):L405-L413.

17. Verkman AS, Galietta LJ. Chloride channels as drug targets. Nat Rev Drug Discov. 2009;8(2):153-171.

18. Van Goor F, Yu H, Burton B, Hoffman BJ. Effect of ivacaftor on CFTR forms with missense mutations associated with defects in protein processing or function. $J$ Cyst Fibros. 2014;13(1):29-36.

19. Yu H, et al. Ivacaftor potentiation of multiple CFTR channels with gating mutations. J Cyst Fibros. 2012;11(3):237-245.

20. Durmowicz T, Pacanowski M. Novel Approach Allows Expansion of Indication for Cystic Fibrosis Drug. FDA. https://www. fda.gov/Drugs/NewsEvents/ucm559051.htm. Updated May 18, 2017 Accessed June 28, 2019.

21. Gottschalk LB, et al. Creation and characterization of an airway epithelial cell line for stable expression of CFTR variants J Cyst Fibros. 2016;15(3):285-294.

22. Raraigh KS, et al. Functional Assays Are Essential for Interpretation of Missense Variants Associated with Variable Expressivity. Am J Hum Genet. 2018;102(6):1062-1077.

23. Kristidis P, et al. Genetic determination of exocrine pancreatic function in cystic fibrosis. Am J Hum Genet. 1992;50(6):1178-1184.

24. Sheppard DN, Ostedgaard LS, Winter MC, Welsh MJ. Mechanism of dysfunction of two nucleotide binding domain mutations in cystic fibrosis transmembrane conductance regulator that are associated with pancreatic sufficiency. EMBO J. 1995;14(5):876-883.

25. Bruscia E, Sangiuolo F, Sinibaldi P, Goncz KK, Novelli G, Gruenert DC. Isolation of CF cell lines corrected at DeltaF508-CFTR locus by SFHR-mediated targeting. Gene Ther. 2002;9(11):683-685.

26. Hong JS. Epithelial Models Encoding Diverse CFTR-2 Mutations for Studies of Disease Mechanism And Drug Discovery. Presented at: The 28th Annual North American Cystic Fibrosis Conference Georgia World Congress Center; Atlanta, Georgia, USA, October 9, 2014. Abstract 175. https://onlinelibrary.wiley.com/doi/abs/10.1002/ppul.23105. Accessed July 12, 2018.

27. Ma T, et al. Thiazolidinone CFTR inhibitor identified by high-throughput screening blocks cholera toxin-induced intestinal fluid secretion. J Clin Invest. 2002;110(11):1651-1658.

28. Ramsey BW, et al. A CFTR potentiator in patients with cystic fibrosis and the G551D mutation. N Engl J Med. 2011;365(18):1663-1672.

29. Cai Z, Taddei A, Sheppard DN. Differential sensitivity of the cystic fibrosis (CF)-associated mutants G551D and G1349D to potentiators of the cystic fibrosis transmembrane conductance regulator (CFTR) Cl- channel. J Biol Chem. 2006;281(4):1970-1977.

30. Bompadre SG, Sohma Y, Li M, Hwang TC. G551D and G1349D, two CF-associated mutations in the signature sequences of CFTR, exhibit distinct gating defects. J Gen Physiol. 2007;129(4):285-298.

31. Sheppard DN, Rich DP, Ostedgaard LS, Gregory RJ, Smith AE, Welsh MJ. Mutations in CFTR associated with mild-diseaseform Cl- channels with altered pore properties. Nature. 1993;362(6416):160-164.

32. Dalton J, Kalid O, Schushan M, Ben-Tal N, Villà-Freixa J. New model of cystic fibrosis transmembrane conductance regulator proposes active channel-like conformation. J Chem Inf Model. 2012;52(7):1842-1853. 
33. Corradi V, Vergani P, Tieleman DP. Cystic Fibrosis Transmembrane Conductance Regulator (CFTR): CLOSED AND OPEN STATE CHANNEL MODELS. J Biol Chem. 2015;290(38):22891-22906.

34. Callebaut I, Hoffmann B, Lehn P, Mornon JP. Molecular modelling and molecular dynamics of CFTR. Cell Mol Life Sci. 2017;74(1):3-22.

35. Zhang Z, Chen J. Atomic Structure of the Cystic Fibrosis Transmembrane Conductance Regulator. Cell. 2016;167(6):1586-1597.e9. 36. Liu F, Zhang Z, Csanády L, Gadsby DC, Chen J. Molecular Structure of the Human CFTR Ion Channel. Cell. 2017;169(1):85-95.e8 37. Zhang Z, Liu F, Chen J. Conformational Changes of CFTR upon Phosphorylation and ATP Binding. Cell. 2017;170(3):483-491.e8. 38. Tabcharani JA, et al. Multi-ion pore behaviour in the CFTR chloride channel. Nature. 1993;366(6450):79-82.

39. Linsdell P, Zheng SX, Hanrahan JW. Non-pore lining amino acid side chains influence anion selectivity of the human CFTR Cl- channel expressed in mammalian cell lines. J Physiol (Lond). 1998;512(Pt 1):1-16.

40. Linsdell P. Relationship between anion binding and anion permeability revealed by mutagenesis within the cystic fibrosis transmembrane conductance regulator chloride channel pore. J Physiol (Lond). 2001;531(Pt 1):51-66.

41. McDonough S, Davidson N, Lester HA, McCarty NA. Novel pore-lining residues in CFTR that govern permeation and open-channel block. Neuron. 1994;13(3):623-634.

42. Bai Y, Li M, Hwang TC. Dual roles of the sixth transmembrane segment of the CFTR chloride channel in gating and permeation. J Gen Physiol. 2010;136(3):293-309.

43. Alexander $\mathrm{C}$, et al. Cystic fibrosis transmembrane conductance regulator: using differential reactivity toward channel-permeant and channel-impermeant thiol-reactive probes to test a molecular model for the pore. Biochemistry. 2009;48(42):10078-10088.

44. Norimatsu Y, et al. Locating a plausible binding site for an open-channel blocker, GlyH-101, in the pore of the cystic fibrosis transmembrane conductance regulator. Mol Pharmacol. 2012;82(6):1042-1055.

45. Okiyoneda T, et al. Mechanism-based corrector combination restores $\triangle$ F508-CFTR folding and function. Nat Chem Biol. 2013;9(7):444-454.

46. Sabusap CM, et al. Analysis of cystic fibrosis-associated P67L CFTR illustrates barriers to personalized therapeutics for orphan diseases. JCI Insight. 2016;1(14):e86581.

47. Ren HY, et al. VX-809 corrects folding defects in cystic fibrosis transmembrane conductance regulator protein through action on membrane-spanning domain 1. Mol Biol Cell. 2013;24(19):3016-3024.

48. Hudson RP, et al. Direct Binding of the Corrector VX-809 to Human CFTR NBD1: Evidence of an Allosteric Coupling between the Binding Site and the NBD1:CL4 Interface. Mol Pharmacol. 2017;92(2):124-135.

49. Loo TW, Bartlett MC, Clarke DM. Corrector VX-809 stabilizes the first transmembrane domain of CFTR. Biochem Pharmacol 2013;86(5):612-619

50. Yousef S, Solomon GM, Brody A, Rowe SM, Colin AA. Improved clinical and radiographic outcomes after treatment with ivacaftor in a young adult with cystic fibrosis with the P67L CFTR mutation. Chest. 2015;147(3):e79-e82.

51. Guigui S, Wang J, Cohen RI. The use of ivacaftor in CFTR mutations resulting in residual functioning protein. Respir Med Case Rep. 2016;19:193-195

52. Cutting GR. Treating Specific Variants Causing Cystic Fibrosis. JAMA. 2017;318(21):2130-2131.

53. Chen JM, et al. A combined analysis of the cystic fibrosis transmembrane conductance regulator: implications for structure and disease models. Mol Biol Evol. 2001;18(9):1771-1788.

54. Jih KY, Lin WY, Sohma Y, Hwang TC. CFTR potentiators: from bench to bedside. Curr Opin Pharmacol. 2017;34:98-104.

55. Graeber SY, et al. Effects of Lumacaftor-Ivacaftor Therapy on Cystic Fibrosis Transmembrane Conductance Regulator Function in Phe508del Homozygous Patients with Cystic Fibrosis. Am J Respir Crit Care Med. 2018;197(11):1433-1442.

56. Pranke IM, et al. Correction of CFTR function in nasal epithelial cells from cystic fibrosis patients predicts improvement of respiratory function by CFTR modulators. Sci Rep. 2017;7(1):7375.

57. Avramescu RG, et al. Mutation-specific downregulation of CFTR2 variants by gating potentiators. Hum Mol Genet. 2017;26(24):4873-4885.

58. Pettit RS, Fellner C. CFTR Modulators for the Treatment of Cystic Fibrosis. P T. 2014;39(7):500-511.

59. Loo TW, Clarke DM. Corrector VX-809 promotes interactions between cytoplasmic loop one and the first nucleotide-binding domain of CFTR. Biochem Pharmacol. 2017;136:24-31.

60. Veit G, et al. Some gating potentiators, including VX-770, diminish $\Delta$ F508-CFTR functional expression. Sci Transl Med. 2014;6(246):246ra97.

61. Mendoza JL, et al. Requirements for efficient correction of $\triangle$ F508 CFTR revealed by analyses of evolved sequences. Cell. 2012;148(1-2):164-174

62. Rabeh WM, et al. Correction of both NBD1 energetics and domain interface is required to restore $\triangle$ F508 CFTR folding and function. Cell. 2012;148(1-2):150-163.

63. Mijnders M, Kleizen B, Braakman I. Correcting CFTR folding defects by small-molecule correctors to cure cystic fibrosis. Curr Opin Pharmacol. 2017;34:83-90.

64. Ooi CY, et al. Type of CFTR mutation determines risk of pancreatitis in patients with cystic fibrosis. Gastroenterology. 2011;140(1):153-161.

65. Vanscoy LL, et al. Heritability of lung disease severity in cystic fibrosis. Am J Respir Crit Care Med. 2007;175(10):1036-1043.

66. Ramalho AS, Beck S, Meyer M, Penque D, Cutting GR, Amaral MD. Five percent of normal cystic fibrosis transmembrane conductance regulator mRNA ameliorates the severity of pulmonary disease in cystic fibrosis. Am J Respir Cell Mol Biol. 2002;27(5):619-627.

67. Zhang L, et al. CFTR delivery to $25 \%$ of surface epithelial cells restores normal rates of mucus transport to human cystic fibrosis airway epithelium. PLoS Biol. 2009;7(7):e1000155.

68. Sosnay PR, et al. Defining the disease liability of variants in the cystic fibrosis transmembrane conductance regulator gene. Nat Genet. 2013;45(10):1160-1167.

69. Pedemonte N, et al. Phenylglycine and sulfonamide correctors of defective delta F508 and G551D cystic fibrosis transmembrane conductance regulator chloride-channel gating. Mol Pharmacol. 2005;67(5):1797-1807.

70. Caci E, et al. CFTR activation in human bronchial epithelial cells by novel benzoflavone and benzimidazolone compounds. Am 
J Physiol Lung Cell Mol Physiol. 2003;285(1):L180-L188.

71. Yang H, et al. Nanomolar affinity small molecule correctors of defective Delta F508-CFTR chloride channel gating. $J$ Biol Chem. 2003;278(37):35079-35085.

72. Devor DC, Bridges RJ, Pilewski JM. Pharmacological modulation of ion transport across wild-type and DeltaF508 CFTR-expressing human bronchial epithelia. Am J Physiol, Cell Physiol. 2000;279(2):C461-C479.

73. Springsteel MF, et al. Benzoflavone activators of the cystic fibrosis transmembrane conductance regulator: towards a pharmacophore model for the nucleotide-binding domain. Bioorg Med Chem. 2003;11(18):4113-4120.

74. Pedemonte N, et al. Small-molecule correctors of defective DeltaF508-CFTR cellular processing identified by high-throughput screening. J Clin Invest. 2005;115(9):2564-2571.

75. Loo TW, Bartlett MC, Wang Y, Clarke DM. The chemical chaperone CFcor-325 repairs folding defects in the transmembrane domains of CFTR-processing mutants. Biochem J. 2006;395(3):537-542.

76. Robert R, et al. Structural analog of sildenafil identified as a novel corrector of the F508del-CFTR trafficking defect. Mol Pharmacol. 2008;73(2):478-489.

77. Macia E, Ehrlich M, Massol R, Boucrot E, Brunner C, Kirchhausen T. Dynasore, a cell-permeable inhibitor of dynamin. Dev Cell. 2006;10(6):839-850.

78. Yoo CL, Yu GJ, Yang B, Robins LI, Verkman AS, Kurth MJ. 4'-Methyl-4,5'-bithiazole-based correctors of defective delta F508-CFTR cellular processing. Bioorg Med Chem Lett. 2008;18(8):2610-2614.

79. Hirth BH, et al. Discovery of 1,2,3,4-tetrahydroisoquinoline-3-carboxylic acid diamides that increase CFTR mediated chloride transport. Bioorg Med Chem Lett. 2005;15(8):2087-2091. 